\title{
A new multi-frequency approach based on Padé approximants for the treatment of transient dynamics problems with the variational theory of complex rays
}

\author{
C. Rouzaud ${ }^{1 *}$, F. Gatuingt ${ }^{2}$, G. Hervé ${ }^{1,3}$, O. Dorival ${ }^{4,5}$ \\ ${ }^{1}$ Université Paris-Est, RENON (IRC-ESTP, IFSTTAR), IRC-ESTP, \\ ${ }^{2}$ LMT (ENS Cachan, CNRS, Université Paris Saclay) \\ ${ }^{3}$ IMSIA, UMR 9219 EDF-CNRS-CEA-ENSTA ParisTech, Université Paris Saclay \\ ${ }^{4}$ Icam, site de Toulouse \\ ${ }^{5}$ Université de Toulouse, Institut Clément Ader (ICA), INSA, UPS, Mines Albi, ISAE
}

\begin{abstract}
Frequency-based methods were set up in order to circumvent the limits of classical finite element methods in fast dynamic simulations due to discretizations. In this approach the dynamic loading was shifted in the frequency domain by FFT, then treated by the Variational Theory of Complex Rays, and then the time response was reconstructed through an IFFT. This strategy proved to be very efficient due to the CPU VTCR very low cost. However in the case of a large loading spectrum this frequency-by-frequency approach could seriously degrade the computational performances of the strategy. This paper addresses this point by proposing the use of Padé approximants in order to limit the number of frequencies at which the response should be calculated. Padé approximation is applied to the overall VTCR system based on its frequency dependency. Finally, as simulations on a simple academic case and on a civil engineering structure show, this method is found to be very efficient for interpolating the frequency response functions of a complex structure. This is a key point to preserve the efficiency of the complete VTCR strategy for transient dynamic problems.

Keywords : model reduction, frequency resolution, Padé approximants, VTCR.
\end{abstract}




\section{Nomenclature}

\begin{tabular}{cl}
\hline Parameters & Physical meaning \\
$f$ & frequency $\left(\mathrm{s}^{-1}\right)$ \\
$\omega$ & angular frequency $\left({\left.\mathrm{rad} . \mathrm{s}^{-1}\right)}^{-1}\right)$ \\
$\omega_{0}$ & central pulsation $\left(\mathrm{rad}_{\mathrm{s}}{ }^{-1}\right)$ \\
$\Delta \omega$ & pulsation bandwidth $\left(\mathrm{rad}_{\mathrm{s}} \mathrm{s}^{-1}\right)$ \\
$\Omega$ & space domain \\
$\Omega_{i}$ & subdomain $i$ \\
$\Gamma$ & common border between two subdomains \\
$(\mathbf{x}, \mathbf{y}, \mathbf{z})$ & cartesian basis vectors \\
$\left(\mathbf{e}_{\alpha}, \mathbf{e}_{\beta}, \mathbf{e}_{\mathbf{3}}\right)$ & local base tangent to the shell in a given point \\
$\mathbf{r}(\alpha, \beta)$ & position vector \\
$\partial \Omega_{i}$ & prescribed boundary \\
$\mathbf{n}, \mathbf{t}$ & normal and tangent vectors to the edge of the subdomain \\
$\mathbf{U}$ & displacement of the average surface \\
$\mathbf{u}$ & membrane displacement \\
$w$ & out-of-plane displacement \\
$\theta$ & rotation \\
$\sigma$ & stress in the average surface \\
$K$ & out-of-plane forces \\
$\mathbf{N}$ & membrane forces \\
$\mathcal{M}$ & out-of-plane momentum \\
$h$ & shell thickness \\
$\rho$ & density \\
$\eta$ & structural damping coefficient \\
$\mathcal{K}_{C P}$ & Hooke's operator in plane stress \\
$E$ & Young's modulus \\
$\nu$ & Poisson's ratio \\
$S_{a d}$ & approximation basis subspace \\
$\mathbf{U}^{h}, \sigma^{h}$ & approximated value of displacements and stresses \\
$\mathbf{U}_{\mathbf{n}}, \mathcal{C}_{n}$ & amplitudes of the local vibration waves in displacements and stresses \\
$\mathbf{Z}$ & position vector \\
$\mathbf{P}$ & vector characterizing the direction of local vibration waves \\
$\varphi$ & directions of the waves \\
$C$ & circle for the directions of the waves \\
$c_{\mathrm{sv}}$ & celerity of bending waves \\
$c_{\mathrm{p}}$ & celerity of pressure waves \\
$c_{\mathrm{sh}}$ & celerity of shear waves \\
$\mathbb{A}$ & VTCR bilinear operator in equation $(4)$ \\
$\mathbf{A}$ & matrix form of $\mathbb{A}$ \\
$\mathbb{L}$ & VTCR linear operator in equation (5) \\
& \\
\hline &
\end{tabular}




$\begin{array}{cl}\mathbf{B} & \text { vector form of } \mathbb{L} \\ \mathbf{X} & \text { amplitudes vector of the wave functions } \\ T & \text { truncation order } \\ \chi & \text { coefficient vector } \\ N & \text { number of degrees of freedom, or the number of wave functions used } \\ L, M & \text { truncated orders } \\ P_{L}, Q_{M} & \text { Taylor series truncated respectively to order } L \text { and } M \\ p, q & \text { Taylor series coefficients } \\ x & \text { coefficients for the Taylor serie } X \\ \mathcal{A} & \text { matrix of Padé coefficients }\end{array}$




\section{Introduction}

When a projectile impacts a structure, several wave regimes occur that depend on the observation time and the structure properties and geometry. Classically these wave regimes can be divided into two types: tension-compression waves and flexural waves which propagate longitudinally. The first type is non-dispersive and mainly affects the damages in the impacted area, while the second one is dispersive and has a global effect on the structure.

The vibratory response of elastic structures in the low-frequency range is not a problem even for complex structures and for the high-frequency range, it exists efficient numerical tools mainly based on energetic approaches.

In this work we are interested in the effect of an aircraft impact on large civil engineering industrial structures. This type of load covers a large frequency range, including the medium frequency range $[1,2]$. The cut-off frequency for this type of load is typically within the 50-100 Hz range, which will be referred to as the medium frequency range for civil engineering structures [3]. Due to the complexity of the problem, it is impossible to use simplified approaches, as in [4], based on spring-mass more or less complex models. The finite elements method with a global model seems to be the most appropriate choice in our case. In order to properly describe the response of the impact area, the finite element method requires that space and time discretizations are sufficiently refined. Ten linear elements per wavelength if often used as a rule of thumb [5, 6]. Time step is limited by the Courant condition for explicit time integration scheme used for this kind of simulations, so that the duration for a wave crossing a discrete spatial grid is less than the time for the wave to travel to adjacent grid points. For this reason, computation time quickly becomes prohibitive when entire civil engineering structures need to be calculated under impact loadings. As a consequence of the mesh refinement limitation, the medium frequency range is often ignored in this type of simulation, which alters the loading that the structure undergoes. This may result in wrong predictions from the simulations.

We can find in the literature some methods that can deal with complex structures. They can be based on structural partitioning as in [7], on methods developed for some types of structures [8], on specific finite-element developments [9]. In a previous work [10] we proposed a new way of calculating the shaking of civil engineering structures, that allows to account for medium frequency range. This methodology is based on the use of the Variational Theory of Complex Rays (VTCR) [11, 12, 13, 14], which is a wave-based computational approach dedicated to the resolution of forced vibration problems at a given frequency. The VTCR uses a weak formulation of the boundary conditions that is projected on a space of admissible shape functions that satisfy both the governing equation and the constitutive relation. Such shape functions are found to show a strong vibrational content as they describe classical waves in infinite or semi-infinite domains. In order to address transient dynamic problems, the proposed strategy is to use a shift from the time domain to the frequency domain that is achieved by a Fast Fourier Transform (FFT) technique [15]. After solving the problem in the frequency domain with the VTCR, a time recombination is performed by an inverse Fast Fourier Transform (IFFT). The approach used in [10] can be very efficient because at each given frequency in the loading spectrum, the VTCR performs a fast resolution of the problem. However, when the loading spectrum contains a lot of frequencies, performances of the frequency-by-frequency treatment are decreased dramatically. The same kind of problem is found when the solution of the Helmholtz equation is required over a broad frequency range that can be found in various engineering applications.

The following article proposes an alternate multi-frequency approach based on Padé approximants for improving the reconstruction of the Frequency Response Functions (FRF). Padé approximants technique consists in the approximation of a function by a rational function of given order. The tech- 
nique was developed around 1890 by Henri Padé [16]. The model reduction by Padé 's approximants we have developed is based on its use for acoustic problems given in [17] Using reduced-order models build via Padé 's approximations are now well established for systems exhibiting polynomial frequency dependency of second order kind and for independent frequency excitations. This paper deals with a more complex situation where a more complicated wave number dependency is encountered due to the frequency dependent loading.

The paper is structured as follows: Section 2 presents the reference problem and its solution using the VTCR; Section 3 gives a general presentation of the reconstruction method by Padé approximants; Section 4 illustrates an application of the strategy on a simple example and on an industrial structure. Finally, conclusions and perspectives are drawn in Section 5.

\section{Description of the Variational Theory of Complex Rays for transient dynamics}

In this section, a brief reminder of the time-frequency strategy (see Figure 1) and of the VTCR methodology are given.

\subsection{Time-to-frequency-to-time domain strategy}

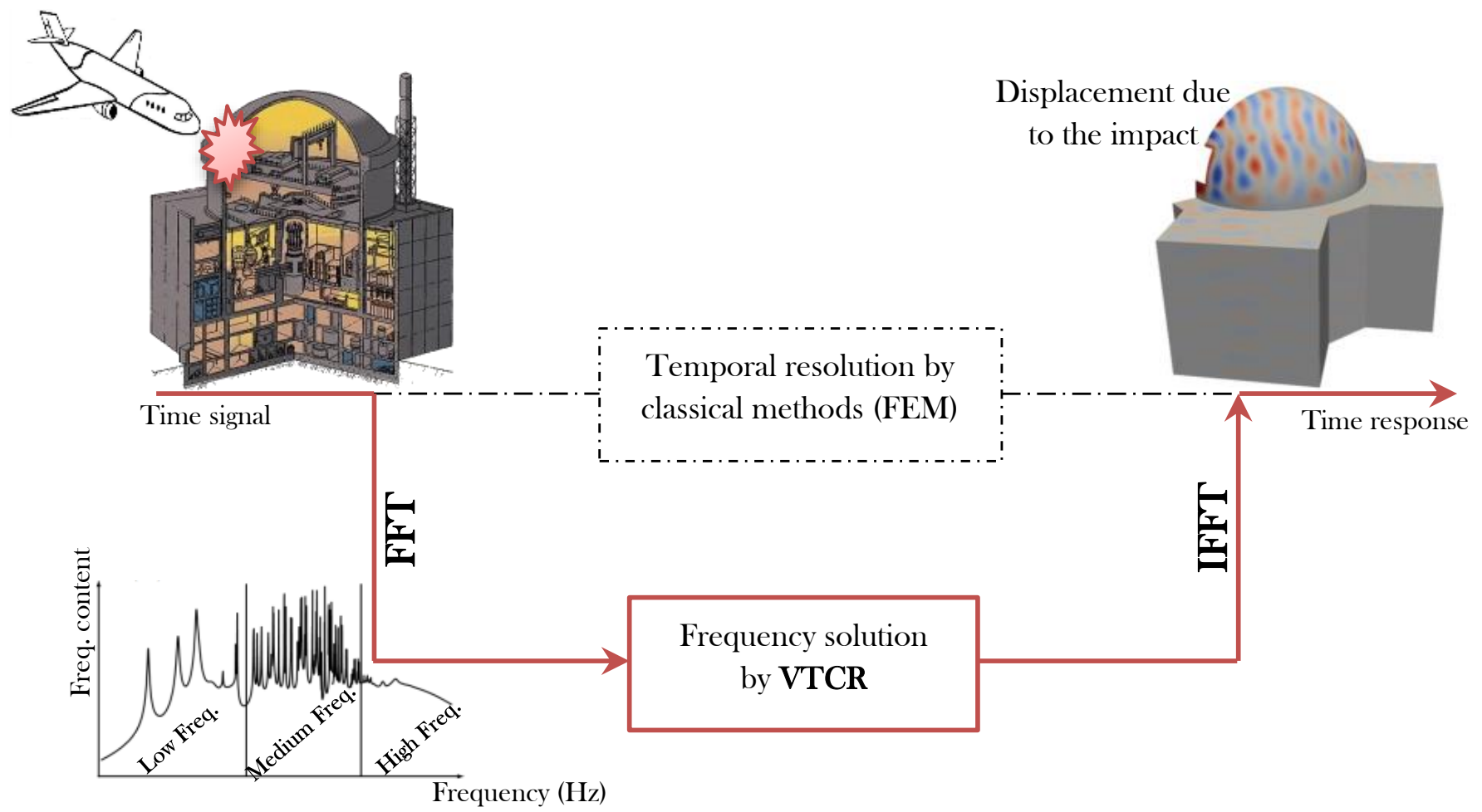

Figure 1: The time-frequency strategy.

The Variational Theory of Complex Rays (VTCR) [12, 15] allows to handle low and medium frequency ranges. High frequencies, which are known to vanish quickly and/or which do not contain a lot of energy, are neglected [18]. 


\subsection{The reference problem at a given frequency for an assembly of two substructures}

Let us consider the case of homogeneous Kirchhoff-Love's thin plates $\Omega_{i}$ which vibrate at a fixed frequency $f=\frac{\omega}{2 \pi}$. The thickness is denoted by $h_{i}$ and the density $\rho_{i}$. Under the Kirchhoff-Love assumptions, the displacement $\mathbf{U}_{i}$ of the average surface becomes:

$$
\begin{gathered}
\mathbf{U}_{i}(\mathbf{x}, \mathbf{y}, \mathbf{z})=\mathbf{u}_{i}(\mathbf{x}, \mathbf{y})+w_{i}(\mathbf{x}, \mathbf{y}) \mathbf{e}_{\mathbf{3}}+\mathbf{z} \theta_{i} \\
\theta_{i}(\mathbf{x}, \mathbf{y})=-\operatorname{grad} w_{i}(\mathbf{x}, \mathbf{y})
\end{gathered}
$$

where $\mathbf{u}_{i}$ is the displacement of the average surface and $w_{i}$ is the out-of-plane displacement. The average surface of the plate can be defined by two independent parameters $\alpha_{i}$ and $\beta_{i}$. The location of a point on the medium surface is defined by the position vector $\mathbf{r}_{i}\left(\alpha_{i}, \beta_{i}\right)$ (see Figure 2).

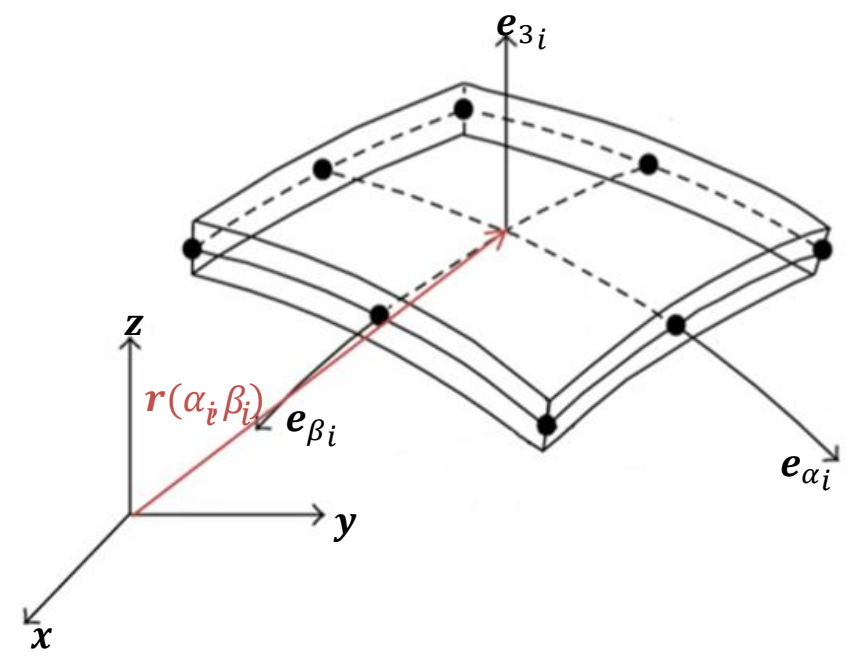

Figure 2: Geometry of a plate $\Omega_{i}$.

The tangent local plane to the plate at point $\mathbf{r}_{i}\left(\alpha_{i}, \beta_{i}\right)$ is defined by two vectors, $\frac{\partial \mathbf{r}_{i}}{\partial \alpha_{i}}=A_{i} \mathbf{e}_{\alpha i}$ and $\frac{\partial \mathbf{r}_{i}}{\partial \beta_{i}}=B_{i} \mathbf{e}_{\beta_{i}}$ (the vectors $\mathbf{e}_{\alpha i}$ and $\mathbf{e}_{\beta_{i}}$ are unit standards). The vector $\mathbf{e}_{\mathbf{3}_{i}}$ is defined by $\mathbf{e}_{\mathbf{3}_{i}}=\mathbf{e}_{\alpha i} \wedge \mathbf{e}_{\beta_{i}}$. The base $\left(\mathbf{e}_{\alpha i}, \mathbf{e}_{\beta_{i}}, \mathbf{e}_{3 i}\right)$ is then orthogonal.

Let us consider a domain $\Omega$ composed of two plates $\Omega_{i}$ and $\Omega_{j}$ with a common border $\Gamma_{i j}$. The actions of the environment are modeled on $\Omega_{i / j}$ by displacement prescribed on $\partial_{\mathbf{U}} \Omega_{i / j}$ and prescribed stresses on $\partial_{\mathbf{F}} \Omega_{i / j}$.

Figure 3 illustrates these actions on an assembly of plates $\Omega_{i}$ and $\Omega_{j}$.

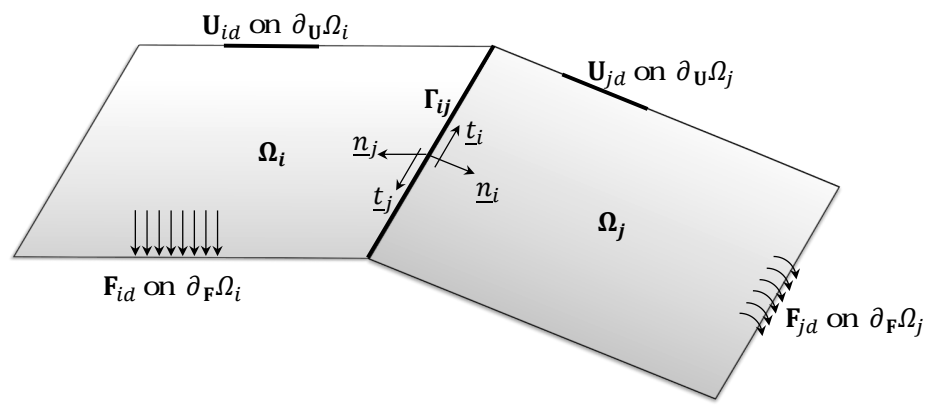

Figure 3: The reference problem. 
In elasticity , $\mathcal{K}_{C P_{i}}$ are Hooke's operators related to each area, under plane stress assumptions; they are defined as:

$$
\mathcal{K}_{C P_{i}}=\left(1+\mathrm{i} \eta_{i}\right) \mathcal{K}_{C P}^{0 i}=\left(1+\mathrm{i} \eta_{i}\right)\left[\begin{array}{ccc}
\frac{E_{\alpha i}}{1-\nu_{\alpha i} \nu_{\beta i}} & \frac{\nu_{\alpha i} E_{\alpha i}}{1-\nu_{\alpha i} \nu_{\beta i}} & 0 \\
\frac{\nu_{\beta i} E_{\beta i}}{1-\nu_{\alpha i} \nu_{\beta i}} & \frac{E_{\beta i}}{1-\nu_{\alpha i} \nu_{\beta i}} & 0 \\
0 & 0 & \frac{\sqrt{E_{\alpha i} E_{\beta i}}}{2\left(1+\sqrt{\nu_{\alpha i} \nu_{\beta i}}\right)}
\end{array}\right]_{\left(\mathbf{e}_{\alpha i}, \mathbf{e}_{\beta i}, \mathbf{e}_{3 i}\right)}
$$

where $\mathrm{i}$ is the imaginary unit, $E_{\alpha, \beta i}$ is the Young's modulus, $\nu_{\alpha, \beta i}$ are the Poisson's ratio in $\mathbf{e}_{\alpha i}$ and $\mathbf{e}_{\beta_{i}}$ directions, $h_{i}$ is the plate thickness, $\rho_{i}$ is the density and $\eta_{i}$ is the structural damping coefficient of each sub-domain $i$.

\subsection{The variational formulation associated with the VTCR}

The first ingredient of the VTCR is a global weak formulation of the boundary conditions in terms of both displacements and forces. The weak formulation can be expressed as follows: find $\left(\mathbf{U}_{i}, \sigma_{i}\right) \in S_{a d, i}$ such as:

$$
\mathbb{A}\left(\left|\begin{array}{l}
s_{i} \\
s_{j}
\end{array},\right| \begin{array}{l}
\delta s_{i} \\
\delta s_{j}
\end{array}\right)=\mathbb{L}\left(\begin{array}{c}
\delta s_{i} \\
\delta s_{j}
\end{array}\right)
$$

with the following general forms:

$$
\begin{aligned}
& \mathbb{A}\left(\begin{array}{c|c}
s_{i} \\
s_{j}
\end{array}, \mid \begin{array}{l}
\delta s_{i} \\
\delta s_{j}
\end{array}\right)=\operatorname{Re}\left\{-\mathrm{i} \omega\left[\int_{\partial_{\mathbf{U}} \Omega_{i}} \delta \sigma_{i} \cdot \mathbf{n}_{i} \cdot \mathbf{U}_{i}^{*} \mathrm{~d} \mathcal{S}+\int_{\partial_{\mathbf{F}} \Omega_{i}} \sigma_{i} \cdot \mathbf{n}_{1} \cdot \delta \mathbf{U}_{i}^{*} \mathrm{~d} \mathcal{S}\right.\right. \\
& +\int_{\partial_{\mathbf{U}} \Omega_{j}} \delta \sigma_{j} \cdot \mathbf{n}_{j} \cdot \mathbf{U}_{j}^{*} \mathrm{~d} \mathcal{S}+\int_{\partial_{\mathbf{F}} \Omega_{j}} \sigma_{j} \cdot \mathbf{n}_{j} \cdot \delta \mathbf{U}_{j}^{*} \mathrm{~d} \mathcal{S} \\
& \left.\left.+\frac{1}{2} \int_{\Gamma_{i j}}\left\{\begin{array}{c}
\left(\delta \sigma_{i} \cdot \mathbf{n}_{i}+\sigma_{j} \cdot \mathbf{n}_{j}\right) \cdot\left(\mathbf{U}_{i}+\mathbf{U}_{j}\right)^{*} \\
+\left(\sigma_{i} \cdot \mathbf{n}_{i}-\sigma_{j} \cdot \mathbf{n}_{j}\right) \cdot\left(\delta \mathbf{U}_{i}-\delta \mathbf{U}_{j}\right)^{*}
\end{array}\right\} \mathrm{d} \mathcal{S}\right]\right\} \\
& \mathbb{L}\left(\mid \begin{array}{l}
\delta s_{i} \\
\delta s_{j}
\end{array}\right)=\operatorname{Re}\left\{-\mathrm{i} \omega\left[\int_{\partial_{\mathbf{U}} \Omega_{i}} \delta \sigma_{i} \cdot \mathbf{n}_{i} \cdot \mathbf{U}_{i d}^{*} \mathrm{~d} \mathcal{S}+\int_{\partial_{\mathbf{F}} \Omega_{i}} \mathbf{F}_{i d} . \delta \mathbf{U}_{i}^{*} \mathrm{~d} \mathcal{S}\right.\right. \\
& \left.\left.+\int_{\partial_{\mathbf{U}} \Omega_{j}} \delta \sigma_{j} \cdot \mathbf{n}_{j} \cdot \mathbf{U}_{j d}^{*} \mathrm{~d} \mathcal{S}+\int_{\partial_{\mathbf{F}} \Omega_{j}} \mathbf{F}_{j d} . \delta \mathbf{U}_{j}^{*} \mathrm{~d} \mathcal{S}\right]\right\}
\end{aligned}
$$

where:

- the integral part on $\partial_{\mathbf{U}} \Omega_{i / j}$ satisfies the prescribed displacements on $\Omega_{i / j}$,

- the integral part on $\partial_{\mathbf{F}} \Omega_{i / j}$ satisfies the prescribed stresses on $\Omega_{i / j}$,

- the integral part on $\Gamma_{i j}$ satisfies the transmission conditions on the boundary $\Gamma_{i j}$.

Re is the real part of a complexe quantity and ${ }^{*}$ the conjugate part. Spaces $\mathcal{S}_{a d, i / j}$ are the admissible fields associated with homogeneous conditions on the substructure $\Omega_{i / j}$.

The previous weak equation is projected on an approximation basis subspace $\mathcal{S}_{a d, i / j}$ that is composed of shape functions that satisfies exactly both the constitutive relation and the dynamic equilibrium equation. 


\subsection{Derivations of two-scale shape functions and the discretized problem}

The VTCR can be viewed as a Trefftz's method which particularity is the use of Herglotz wave functions to represent the vibrational field. Those functions involve an integral combination of plane waves in all the directions. The solution is assumed to be properly described locally as the superposition of an infinite number of these functions which can be written in the following manner:

$$
\begin{aligned}
& \mathbf{U}_{i}\left(\mathbf{Z}_{i}, \mathbf{P}_{i}\right)=\int_{\mathbf{P}_{i} \in C_{i}} \mathbf{U}_{\mathbf{n} i}\left(\mathbf{P}_{i}\right) \cdot \mathrm{e}^{\mathbf{P}_{i} \cdot \mathbf{Z}_{i}} \mathrm{~d} \mathbf{P}_{i} \quad \text { on } \Omega_{i} \\
& \sigma_{i}\left(\mathbf{Z}_{i}, \mathbf{P}_{i}\right)=\int_{\mathbf{P}_{i} \in C_{i}} \mathcal{C}_{n i}\left(\mathbf{P}_{i}\right) \cdot \mathrm{e}^{\mathbf{P}_{i} \cdot \mathbf{Z}_{i}} \mathrm{~d} \mathbf{P}_{i} \quad \text { on } \Omega_{i}
\end{aligned}
$$

where $\mathbf{Z}_{i}$ denotes the position vector. $\mathbf{U}_{\mathbf{n} i}$ and $\mathcal{C}_{n i}$ contain the amplitudes of the associated local vibration waves. $\mathbf{P}_{i}$ characterizes the direction of local waves and is defined on circle $C_{i}$.

In order for these local waves $\left(\mathbf{U}_{i}, \sigma_{i}\right)$ to be admissible, they must be in $\mathcal{S}_{a d, i}$ and satisfy the constitutive and dynamic equilibrium equations. Combining these two equations leads to some remarkable shape functions (named complex rays) characterized by various expressions of $\mathbf{P}_{i}$. For example in the case of a flexural plate the vibration waves $\mathbf{P}$ are defined as :

$$
\left(\mathbf{P}^{\top} \cdot \mathbf{P}\right)^{4}=\frac{12\left(1-\nu^{2}\right) \rho \omega^{2}}{(1+\mathrm{i} \eta) E h^{2}}\left(\mathbf{P}^{\top} \cdot \mathbf{P}\right)^{2}
$$

Note that the term $e^{\mathbf{P}_{i} \cdot \mathbf{Z}_{i}}$ describes the short scale variation, which is known in a closed form, while $\mathbf{U}_{\mathbf{n} i}$ and $\mathcal{C}_{n i}$ are the long scale variations, which remain the unknown to be determined.

Let us note that admissible space $\mathcal{S}_{a d, i}$ is of infinite dimension since all the directions of propagation $\mathbf{P}_{i}$ are taken into account. To end up with a finite dimension problem that can be solved numerically, one needs to discretize $\mathcal{S}_{a d, i}$ into a finite dimension space $\mathcal{S}_{a d, i}^{h}$.

The displacement of any point of the substructure is generated by a basis of admissible complex waves. The unknowns are the generalized amplitudes $\mathbf{U}_{i}^{h}\left(\mathbf{P}_{i}\right)$ of the basis on $\Omega_{i}$. Accounting for all the directions $\varphi_{i}$ in $C_{i}$ leads to an integral over $C_{i}$.

This integral takes the following form:

$$
\begin{aligned}
\mathbf{U}_{i}^{h}\left(\mathbf{z}_{i}\right) & =\int_{\varphi_{i} \in C_{i}} \mathbf{U}_{\mathbf{n}_{i}}^{h}\left(\varphi_{i}\right) \mathrm{e}^{\mathbf{P}_{i}\left(\varphi_{i}\right) \cdot \mathbf{z}_{i}} \mathrm{~d} \varphi_{i} \\
\sigma_{i}^{h}\left(\mathbf{z}_{i}\right) & =\int_{\varphi_{i} \in C_{i}} \mathcal{C}_{n_{i}}^{h}\left(\varphi_{i}\right) \mathrm{e}^{\mathbf{P}_{i}\left(\varphi_{i}\right) \cdot \mathbf{z}_{i}} \mathrm{~d} \varphi_{i}
\end{aligned}
$$

The integrals in Equation (8) and Equation (9) can be discretized as Dirac's functions and one can consider the approximate amplitude $\mathbf{U}^{h}\left(\mathbf{P}_{i}\left(\varphi_{i}\right)\right)$. The angular distributions of the plane waves for all points in the substructure are assumed to be well-described by this discontinuous angular distribution. 


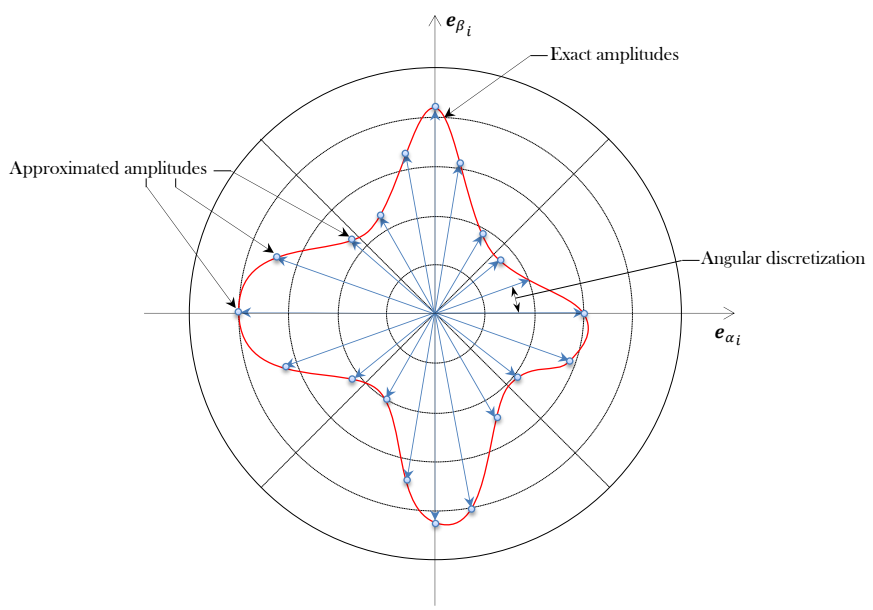

Figure 4: The discretized amplitudes.

The choice of the angular discretization and therefore the number of waves required for solving this kind of problem is related to the number of waves in the structure and on each edge [19].

The number of wavelength $n_{i}$ depends on the celerity of the waves: for pressure waves, $c_{\mathrm{p}}=$ $\sqrt{\frac{E_{\alpha / \beta i}}{\rho_{i}\left(1-\nu_{\alpha i} \nu_{\beta i}\right)}}$, for shear waves, $c_{\mathrm{sh}}=\sqrt{\frac{\sqrt{E_{\alpha i} E_{\beta i}}}{2 \rho_{i}\left(1+\sqrt{\nu_{\alpha i} \nu_{\beta i}}\right)}}$ and for bending waves $c_{\mathrm{sv}}=\sqrt{\omega} \sqrt[4]{\frac{E_{\alpha / \beta i} h_{i}^{3}}{12 \rho_{i} h_{i}\left(1-\nu_{\alpha i} \nu_{\beta i}\right)}}$. form:

Finally at a given frequency $\omega$, the VTCR equation results in a linear problem of the following

$$
\mathbf{A}(\omega) \mathbf{X}(\omega)=\mathbf{B}(\omega)
$$

where $\mathbf{A}$ and $\mathbf{B}$ come from the discretization of operators $\mathbb{A}$ (Eq. 4) and $\mathbb{L}$ (Eq. 5). $\mathbf{X}$ contains the unknown slow scale amplitude of the shape functions. Due to the strong vibrational sense of the shape functions, the VTCR offers a substantial reduction of the size of the problem to solve compared to finite element methods.

\section{A new approach for broadband analysis by the VTCR}

The treatment of an aircraft crash on a civil engineering structure by the strategy proposed in [10] results in a frequency-by-frequency resolution by the VTCR before recombining the solution in the time domain. This may degrade the strategy performances when lots of frequencies need to be accounted for. This article provides an alternate approach based on Padé approximants for the rapid reconstruction of frequency response functions. The aim is to reduce the number of frequencies at which a complete problem must be solved, and then to interpolate the solution between these frequencies. To this aim, a reconstruction based on Padé approximants allows a good complement, involving the calculation of the complete solution and its derivatives for a limited number of frequencies, and a reconstruction around these points. This approach is helped by the expression of the frequency dependency in the form of scalar functions in the overall VTCR equation.

\subsection{Padé approximants}

All VTCR problems, with harmonic vibrations $f=\frac{\omega}{2 \pi}$, have the following form: 


$$
\mathbf{A}(\omega) \mathbf{X}(\omega)=\mathbf{B}(\omega)
$$

In a scheme of direct solution, the calculation of the complete solution of Equation (11) on only few frequency points, coupled to a reconstruction of an approximated solution between them, was found to be an effective approach [20]. Thus, given a function $\mathbf{X}(\omega)$ of $\omega$, the Taylor series development around $\omega_{0}$ gives the following approximate solution:

$$
\mathbf{X}\left(\omega_{0}+\Delta \omega\right) \approx \mathbf{X}_{T}(\Delta \omega)=\sum_{i=0}^{T} \chi_{i}(\Delta \omega)^{i}
$$

$T$ is the truncation order of the Taylor series expansion. The coefficient vector $\chi_{i}$ is given by:

$$
\chi_{i}=\frac{\mathbf{X}^{(i)}\left(\omega_{0}\right)}{i !}
$$

where $\mathbf{X}^{(i)}\left(\omega_{0}\right)$ is the $i^{\text {th }}$ derivative of $\mathbf{X}$ over $\omega$, taking $\omega=\omega_{0}$ and considering that $\mathbf{X}^{(0)}\left(\omega_{0}\right)=\mathbf{X}\left(\omega_{0}\right)$. The determination of the first $T$ derivatives from $\mathbf{X}$ is then required to estimate an approximate solution around $\omega_{0}$.

However, in the context of the series expansion, it has been shown that, for a function with poles as it is the case for structural FRFs, an expansion at a ratio of two power series generally performs a better representation (greater convergence range and better convergence rate) compared to the Taylor series for a given truncation order [21, 22, 23]. In the next part, the approximation at a given frequency $\omega_{0}$ is presented under the methodology for determining Padé approximants of Equation (11). For the sake of clarity, let us consider the $j^{\text {th }}$ component of $\mathbf{X}\left(\omega_{0}\right)$, which is sought around $\omega_{0}$ as :

$$
X_{j}\left(\omega_{0}+\Delta \omega\right) \approx \frac{P_{L j}(\Delta \omega)}{Q_{M j}(\Delta \omega)}
$$

where $P_{L j}(\Delta \omega)$ and $Q_{M j}(\Delta \omega)$ are two Taylor series, truncated respectively to order $L$ and $M$ and defined as:

$$
\begin{aligned}
P_{L j}(\Delta \omega) & =\sum_{i=0}^{L} p_{i j}(\Delta \omega)^{i} \\
Q_{M j}(\Delta \omega) & =\sum_{i=0}^{M} q_{i j}(\Delta \omega)^{i}
\end{aligned}
$$

The approximation of $X_{j}\left(\omega_{0}+\Delta \omega\right)$ is given by the single determination of the coefficients $p_{i j}$ and $q_{i j}$. An efficient approach [22] to determine these coefficients is to identify the coefficients of a Taylor series expansion $X_{L+M j}(\Delta \omega)$ to the order $L+M$ (Equation 12), and so solving the following linear system:

$$
P_{L j}(\Delta \omega)-X_{L+M_{j}}(\Delta \omega) Q_{M_{j}}(\Delta \omega)=0
$$

where the coefficients of order equal to $\Delta \omega$ are extracted to form a set of $(L+M+1)$ equations. With the form of Equation (16), the linear system of equations allows zero for $Q_{M_{j}}(\Delta \omega)$, which makes it more suitable to account for a resonance frequency in the original answer. However, the system of underdetermined Equations (16) gives a solution of $(L+M+2)$ coefficients up to a multiplicative constant. Therefore, they are usually normalized so that the denominator coefficient of the zero order $q_{0 j}$ is taken equal to 1 [22]. In the following, all the equations can then be put as: 


$$
\left.\begin{array}{r}
p_{0 j}=x_{0 j} \\
p_{1 j}-x_{0 j} q_{1_{j}}=x_{1 j} \\
\vdots \\
p_{L j}-x_{L-1 j} q_{1_{j}}-\cdots-x_{0 j} q_{L_{j}}=x_{L j} \\
-x_{L j} q_{1 j}-x_{L-1 j} q_{2 j}-\cdots-x_{L-M+1 j} q_{M j}=x_{L+1 j} \\
\vdots \\
-x_{L+M-1 j} q_{1 j}-x_{L+M-2 j} q_{2 j}-\cdots-x_{L j} q_{M j}=x_{L+M j}
\end{array}\right\}
$$

with

$$
\left\{\begin{array}{l}
x_{i j}=0 \text { if } i<0 \\
q_{i j}=0 \text { if } i>M
\end{array}\right.
$$

Equation (13), gives the expression of the coefficients $x_{i j}$. Equations (17) can be explicitly written by taking into account the $(L+M)$ first derivatives of $X_{j}(\omega)$ to $\omega_{0}$ :

$$
i ! p_{i j}-\left(\sum_{k=1}^{i}\left(\begin{array}{l}
i \\
k
\end{array}\right) k ! X_{j}^{(i-k)}\left(\omega_{0}\right) q_{k j}\right)=X_{j}^{(i)}\left(\omega_{0}\right), \text { for } i=0, \ldots, L+M
$$

where

$$
\left\{\begin{array}{l}
p_{i_{j}}=0 \text { if } i>L \\
q_{k_{j}}=0 \text { if } k>M
\end{array}\right.
$$

and where the binomial coefficient are given by:

$$
\left(\begin{array}{l}
i \\
k
\end{array}\right)=\frac{i !}{k !(i-k) !}
$$

This system can be solved efficiently in the following matrix form for each DoF $j$ involving derivatives up to $L+M$ order of the solution vector :

$$
[\mathcal{A}]_{j}\left[\begin{array}{c}
p_{0} \\
\vdots \\
p_{L} \\
q_{1} \\
\vdots \\
q_{M}
\end{array}\right]_{j}=\left[\begin{array}{c}
X\left(\omega_{0}\right) \\
\vdots \\
X^{(L)}\left(\omega_{0}\right) \\
X^{(L+1)}\left(\omega_{0}\right) \\
\vdots \\
X^{(L+M)}\left(\omega_{0}\right)
\end{array}\right]_{j}, \text { for } j=1, \ldots, N
$$

Based on the solution in a given frequency, $\mathbf{X}\left(\omega_{0}\right)$, this method is defined in the literature as the Padé approximation in a single point. It can easily be extended to several points, as shown for example in [20]. The goal is to estimate the combination of this approach coupled with VTCR. The use of a multipoint Padé approximation is considered as a natural extension of this work. Similarly, an approximation of the solution using the Taylor series expansion, solving Equations (22) requires the determination of the $(L+M)^{t h}$ derivative from the solution vector $\mathbf{X}(\omega)$ in $\omega_{0}$. This is discussed in the next section. 


\subsection{Successive derivatives of the solution vector}

The $(L+M)$ successive derivatives of $\mathbf{X}$ to the pulsation $\omega$ can be obtained by Equation (11). The successive derivatives can be computed by a recursive formula, leading to the solution of a linear system with multiple second members, which is very efficient from a numerical point of view : only one decomposition of the coefficient matrix is to be done. To the order $i$, the expression becomes:

$$
\sum_{k=0}^{i}\left(\begin{array}{l}
i \\
k
\end{array}\right) \mathbf{A}^{(i-k)}\left(\omega_{0}\right) \mathbf{X}^{(k)}\left(\omega_{0}\right)=\mathbf{B}^{(i)}\left(\omega_{0}\right), \text { for } i=1, \ldots,(L+M)
$$

where the zero order derivatives correspond to the undifferentiated functions. Extracting the term of highest order of the sum in Equation (23) leads to the following recursive expression for the $i^{\text {th }}$ order derivative of $\mathbf{X}$ in $\omega_{0}$ :

$$
\mathbf{A}\left(\omega_{0}\right) \mathbf{X}^{(i)}\left(\omega_{0}\right)=\mathbf{B}^{(i)}\left(\omega_{0}\right)-\sum_{k=0}^{i-1}\left(\begin{array}{l}
i \\
k
\end{array}\right) \mathbf{A}^{(i-k)}\left(\omega_{0}\right) \mathbf{X}^{(k)}\left(\omega_{0}\right), \text { for } i=1, \ldots,(L+M)
$$

This implies that the successive derivatives of $\mathbf{X}$ required for the determination of the Padé approximation can be computed efficiently as the solution of a $N$ dimension set of equations. Indeed, the solution can be performed by direct methods using a decomposition of the system matrix. Factorising is then the most numerical consuming step but is processed only one time. Regarding the multiple secondary vectors, they are built from derivatives of the system matrix and derived from lower order vector solution.

\subsection{Procedure for the reconstruction of the frequency response using the Padé approximants}

This section summarizes the calculation steps to approximate the solution over a frequency range using Padé approximants. They can be separated into two parts:

- first, at a given frequency $\omega_{0}$ the solution and its derivatives to successive orders $(L+M)$ are calculated according to Equation (24). This implies the solution of $(L+M+1)$ problems of size $N$ for each frequency at which the solution must be approached;

- then the solution around $\omega_{0}$ is reconstructed, involving solving $N$ problems of $(L+M+1)$ dimensions (see Equation (22)) to determine the Padé approximants for each degree of freedom. Following this, $N$ evaluations of the rational fraction (Equation (14)) are made for each $\Delta \omega$, where the solution evaluated is approached.

Given a discretization of the frequency domain into intervals associated with their center frequency $\omega_{0}$, the detailed procedure corresponding to a frequency range is presented in the algorithm given in Table 1. 
Table 1: Reconstruction algorithm of the response over a frequency range using Padé approximants.

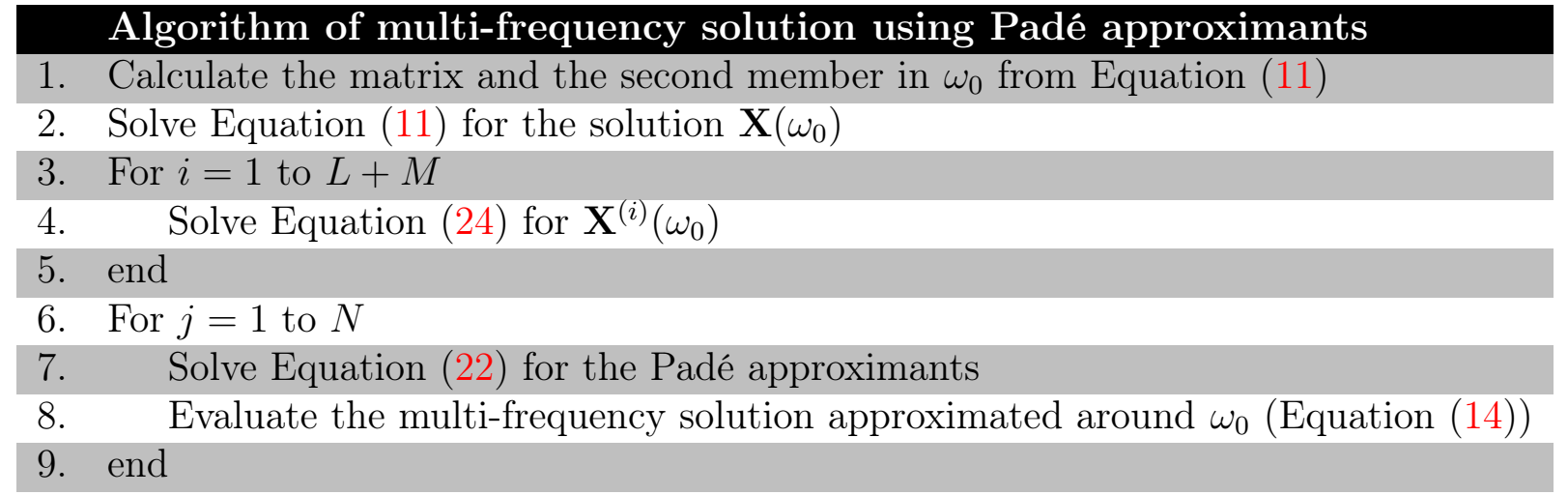

Three points can be considered in view of the given procedure. The first point is to select the secondary frequencies where the solution will be evaluated, or an appropriate discretization of the frequency range. Secondly, for each coarse frequency, the order of the polynomial expansions and frequency range around $\omega_{0}$ should be selected consequently, which raises the question of values of $L$ and $M$. Third, one must evaluate the efficiency conditions for this approximation scheme.

Although the second and third points are discussed in details in [20] for structural or acoustic applications, the choice of secondary frequencies has not received much attention in the state of art. A suggestion is made in [17]. Regarding the order of polynomial expansions to consider for both the numerator $(L)$ and the denominator $(M)$ in Equation (14), the following constraint proposed in [20] reduces the range of possibilities giving satisfying approximations:

$$
M=L+1
$$

In addition, there is an upper limit for the maximum order which can be defined, due to ill conditionning in the resulting matrix system from Equations (22). Therefore, depending on the frequency discretization chosen for a scan of the frequency range, the Padé approximation should be more effective if it could converge on several frequency increments around $\omega_{\max }$. The time allocated to solve several different linear systems of size $N$ leads us to solve $(L+M+1)$ systems with the second member of $N$ equations ( 1 decomposition, and $(L+M+1)$ solutions) and $N$ systems $(L+M+1)$ equations, with $(L+M+1) \ll N$. This methodology has been extremely effective when applied to important structural and acoustic problems with single field and a a priori discretization of the frequency range [24, 20].

\section{Application of multi-frequency analysis}

\subsection{Multi-frequency analysis on a simple case study}

In this study to compare the multi-frequency analysis via Padé approximants with a classical frequencyby-frequency solution, we set ourselves in the case of a bending problem applied to a single KirchhoffLove plate (see Figure 5). 


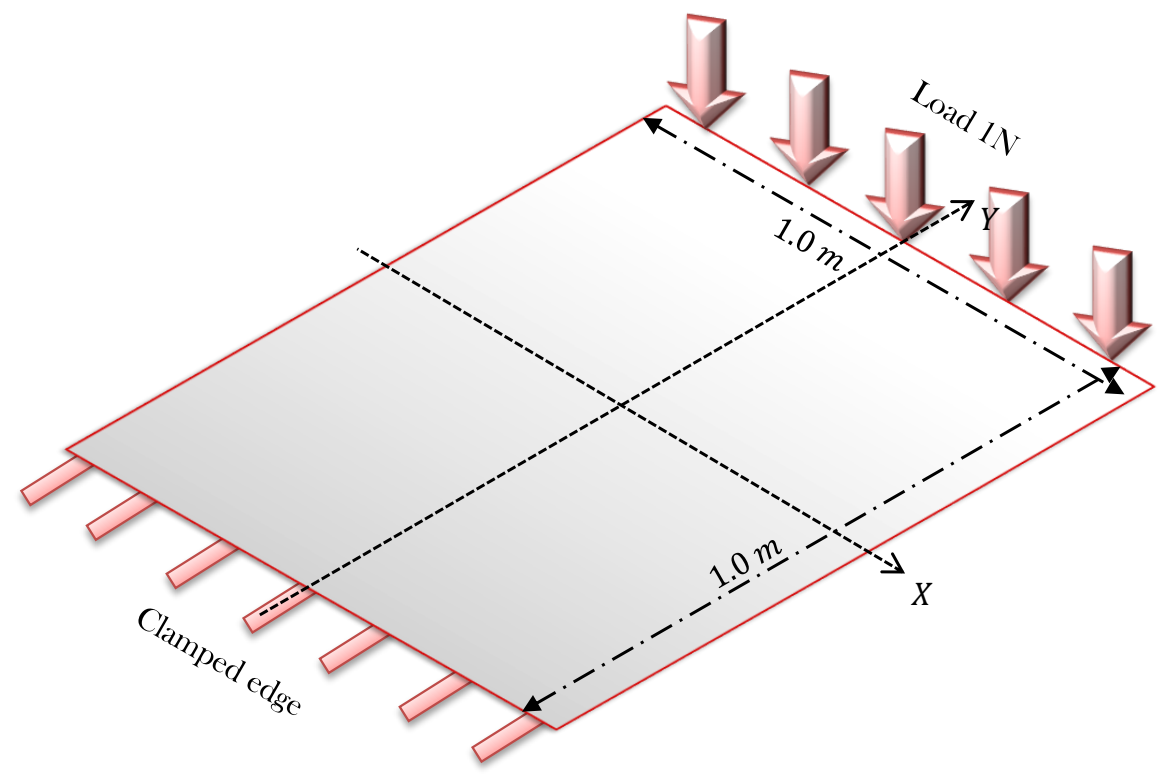

Figure 5: Padé approximants example: description of the geometry and boundary conditions.

The case presented is a plate clamped on one of its edges, free on two others and loaded by a out-ofplane line force on its last edge. The plate is square and has the following dimensions: $1 \mathrm{~m} \times 1 \mathrm{~m} \times$ $0.7 \mathrm{~mm}$. The loaded edge is thus subjected to a unit line force $\mathbf{F}_{d}$ with a direction perpendicular to the mean surface of the plate. The frequency range is $[2 \pi \times 0 \mathrm{~Hz} ; 2 \pi \times 350 \mathrm{~Hz}]$. Materials and structural parameters are given in Table 2.

Table 2: Material and structural parameters of the studied steel plate.

$\begin{array}{ccc}\text { Notation } & \text { Physical quantity } & \text { Value and unit } \\ E & \text { Young's modulus } & 210 \mathrm{GPa} \\ \nu & \text { Poisson's ratio } & 0.3 \\ \rho & \text { mass density } & 7800 \mathrm{~kg} / \mathrm{m}^{3} \\ \xi & \text { damping factor } & 0.01\end{array}$

The comparison between a finite element solutions calculated with Cast3m [25] and the VTCR solution at $\omega=2 \pi \times 150 \mathrm{~Hz}$ is presented in Table 3 .

Table 3: FEM Cast3m solution (left), VTCR solution with 40 DoF (right). 


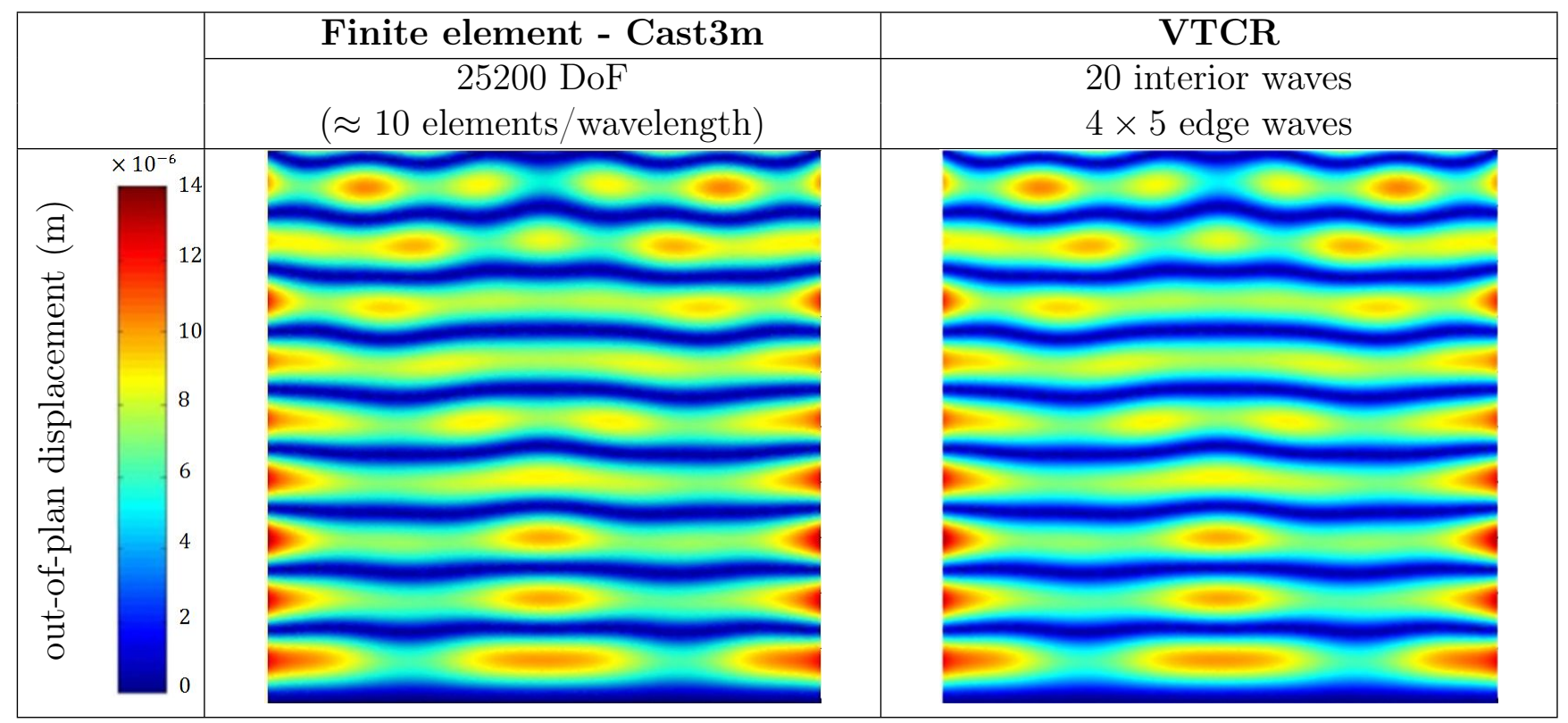

To study the efficiency of multi-frequency solution using Padé approximants, the flexural plate described is studied for a $40 \mathrm{~Hz}$ medium frequency range, from 290 to $330 \mathrm{~Hz}$. In this first study, the goal is to investigate the efficiency of Padé approximants applied to the VTCR problem in terms of both results and calculation time compared to a classical solution at each frequency. This solution repeated each $1 \mathrm{~Hz}$ will be considered as the reference solution. Figure 6 shows the convergence of the frequency response function (FRF) for several sets of parameters $L$ and $M$. Here a single analysis is calculated with $310 \mathrm{~Hz}$ as a center frequency. One can observe that increasing the order $L$ and $M$ of Padé approximants quickly leads to the convergence to the reference solution.

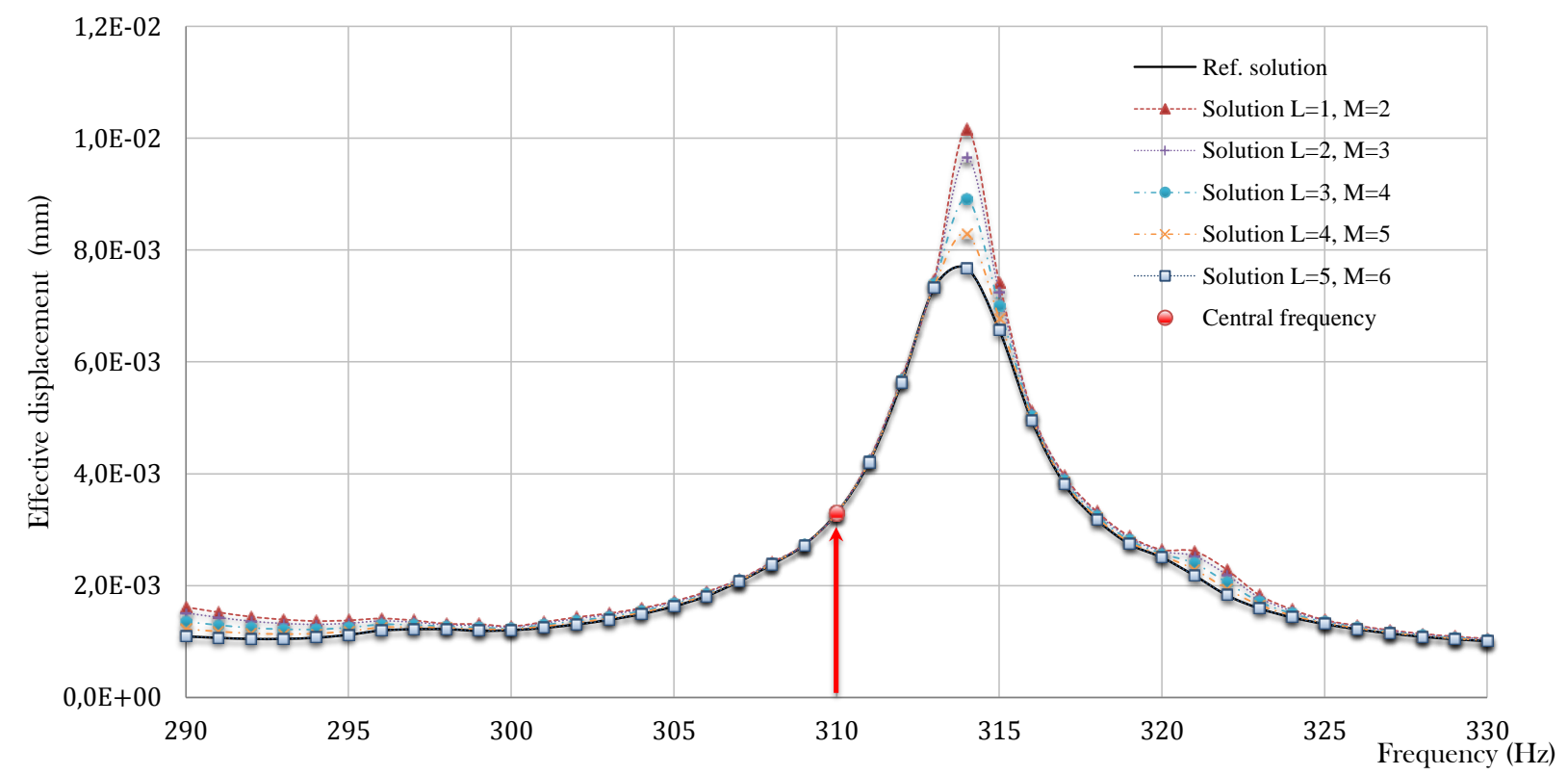

Figure 6: FRF for several sets of parameter $L$ and $M$ over a frequency range of $40 \mathrm{~Hz}$.

Application of Padé approximants to VTCR is found to be efficient when the approximants order is high enough to describe the solution over the frequency range. Nevertheless, if this analysis does not 
require one inversion of the bilinear matrix for the central frequency, it requests to calculate at each frequency the successive derivatives of this matrix and the second member. One can easily imagine that these derivatives may involve significant computational cost if they are not written recursively. This cost also increases with orders $L$ and $M$ as the order of derivation is equal to $L+M$.

Figure 7 compares the computation time for multi-frequency analysis with two sets of parameters and the classical analysis. Note that the approach using Padé approximant is particularly interesting with an important frequency range. Indeed, in the case of a frequency-by-frequency solution, the overall calculation time for $40 \mathrm{~Hz}$ is $95 \mathrm{~s}$, and includes assembly and solution times required for each frequency. For the multi-frequency analysis, the computation time is $47 \mathrm{~s}$ for orders $L=1$ and $M=2$, and $65 \mathrm{~s}$ for orders $L=5$ and $M=6$. In these two cases, a high proportion of the calculation time is dedicated to the construction of the successive derivatives.

Indeed, in this study, the matrix to invert is rather of small size as we treat only one subdomain. Thus, the matrix inversion in the case of a problem with a few plate structures is numerically inexpensive. However, for problems involving a larger number of subdomains, the inversion time will be much higher, and so the analysis via Padé approximants can be expected to be more efficient. 

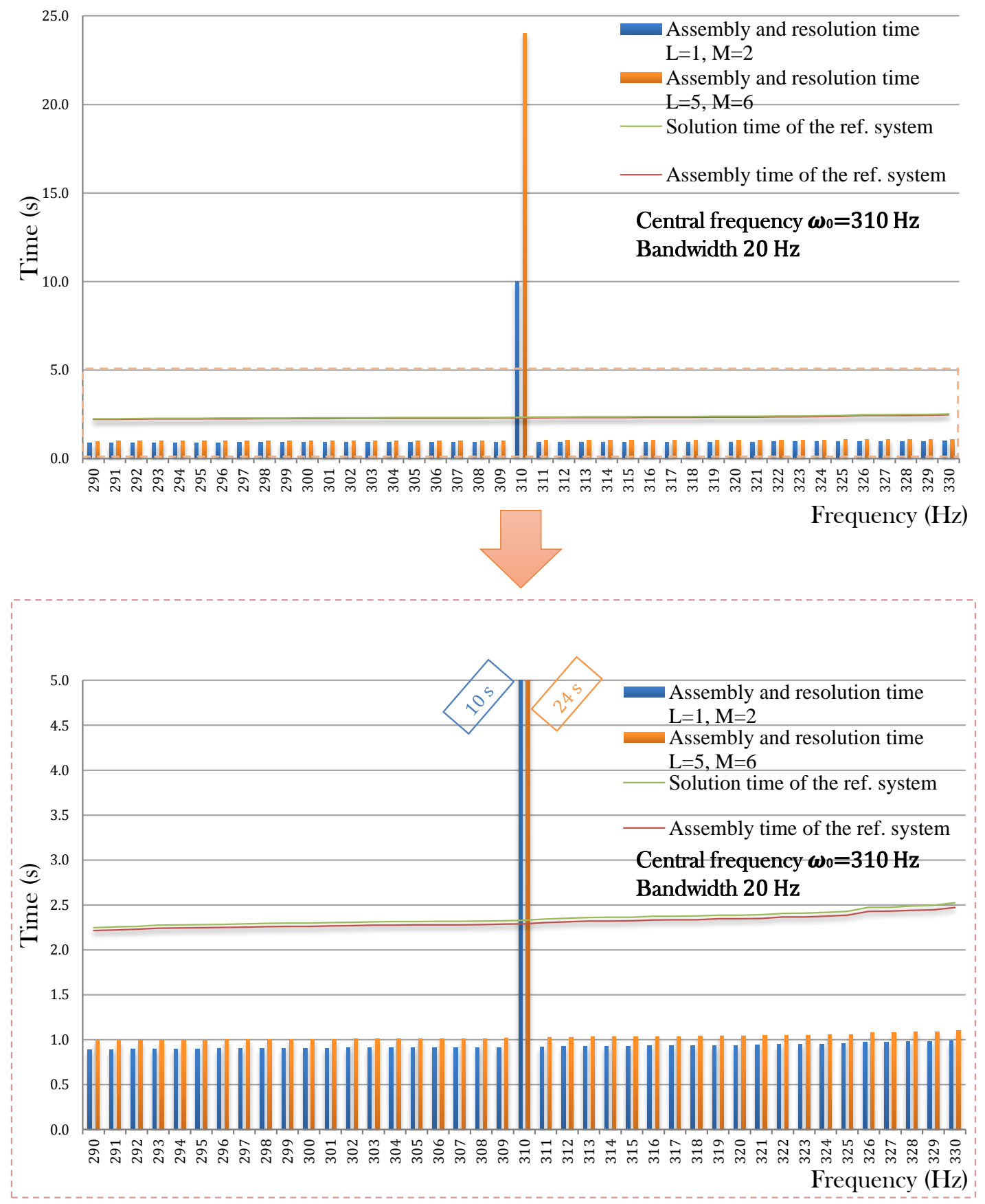

Figure 7: Comparison of computation time for both types of analysis.

Figures 8 and 9 define the FRF calculated over the full frequency range from 0.1 to $350 \mathrm{~Hz}$. Here Padé approximants orders of $L=4$ and $M=5$ were chosen. This parameter set presents the best error/computation time ratio on a small frequency range. A study on the discretization analysis is presented here. This discretization can be observed through the center frequencies chosen to represent the FRF solution. First, in Figure 8, the choice of bandwidth is fixed at $20 \mathrm{~Hz}$ and therefore defined a priori. 


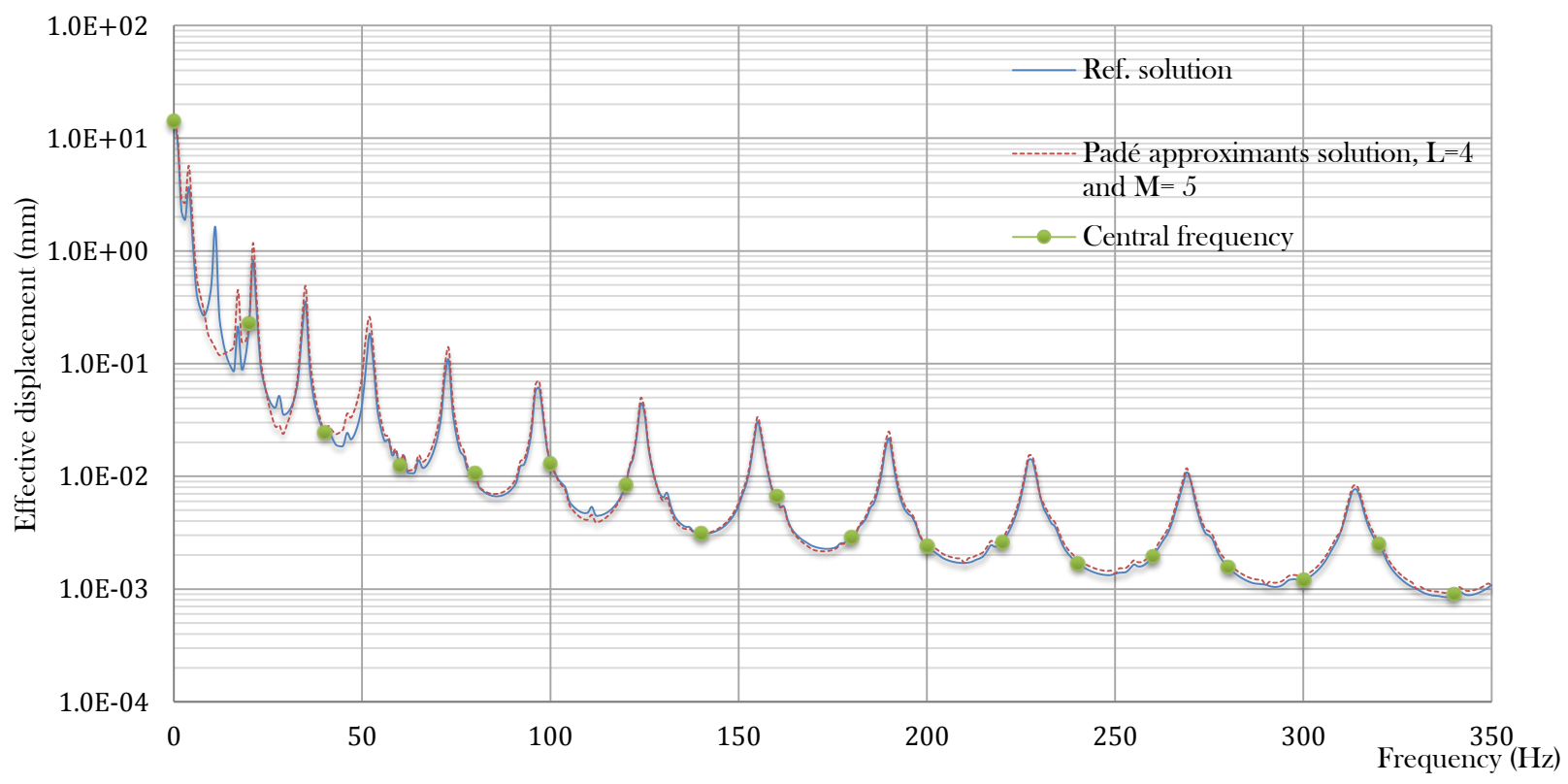

Figure 8: FRF over a wide frequency range with a bandwidth of $20 \mathrm{~Hz}$.

Figure 9 presents a a posteriori study where the bandwidth $\Delta \omega$ increases with respect to the central frequency $\omega_{0}$. The criterion is set so that $\frac{\Delta \omega}{\omega_{0}}<10 \%$. This bandwidth begins at $3 \mathrm{~Hz}$ to finish at 20 Hz. It can be noted that it can induce significant errors when the number of modes per frequency band becomes too important.

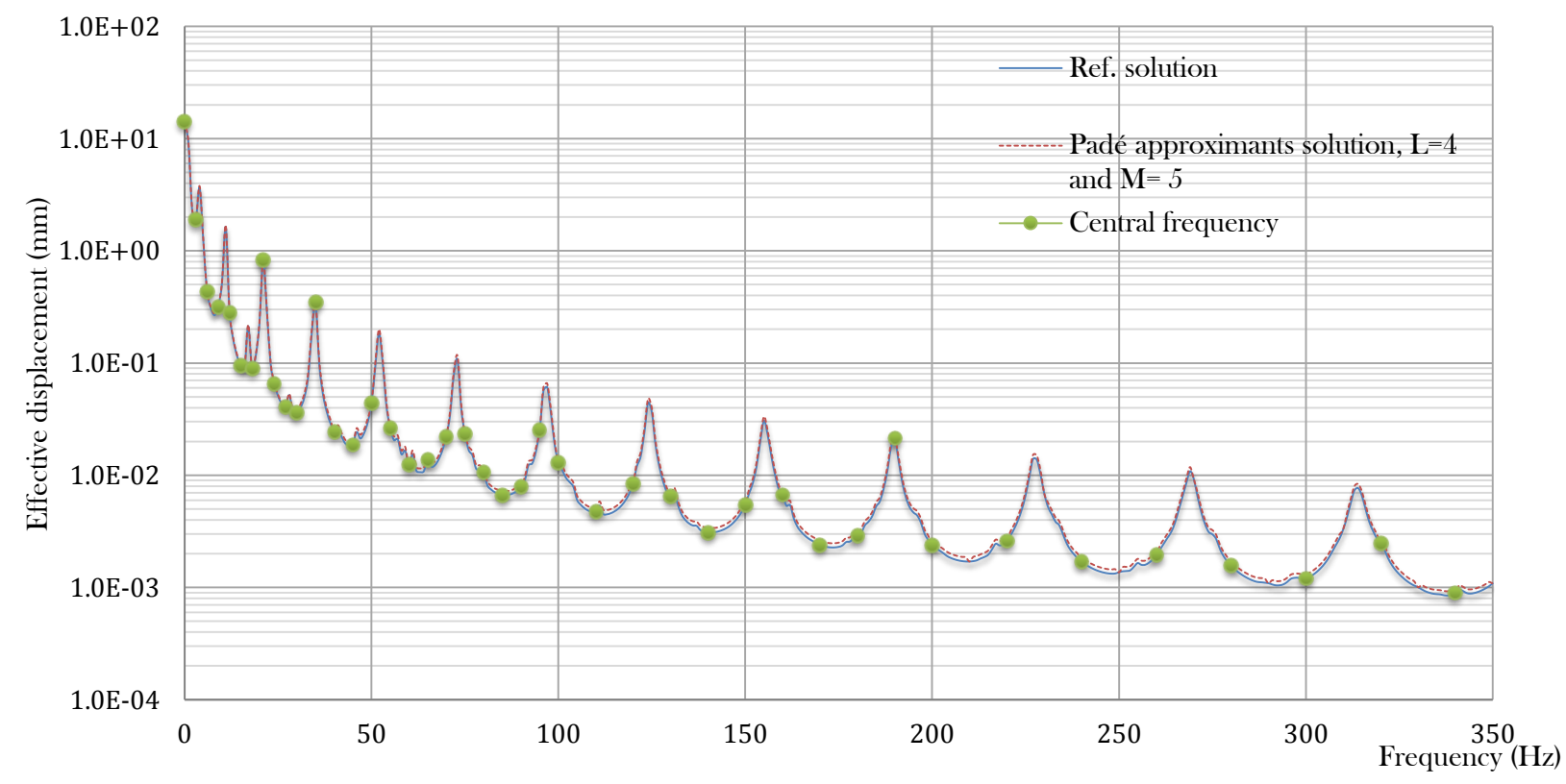

Figure 9: FRF over a wide frequency band, with a standard $10 \%$ criterion.

From this first example one can therefore conclude that the choice of the discretization analysis is 
dependent on the frequency content that one seeks to represent. To solve this, an analysis where the frequency ranges are define by dichotomy from an error criterion can be used.

\subsection{Numerical application of the method on a civil engineering structure}

In this section the VTCR coupled with Padé's multi-frequency approach is used to calculate the frequency response of a structure subjected to a time versine loading. Experimental shaking or vibration studies on large civil engineering structures are lacking in actual literature. For that reason, the comparisons between the VTCR-Padé strategy and the classical explicit time finite element approach is here based on international rules [26] for the design of nuclear civil engineering structures. The comparison between simple VTCR (frequency-by-frequency analysis) and FEM calculation for this structure is given in [10].

The load applied on the civil engineering structure sketched in Figure 10 may be seen as an impulsive load. First the discrete Fourier transform of the load is calculated. Then the frequency response at two chosen points $(P 1)$ and $(P 2)$ of the structure (see in Figure 10) is given by the VTCR.

A concrete structure with a thickness of $1.0 \mathrm{~m}$ is considered where the mechanical properties of concrete are calculated according to the Eurocode 2 rules:

- concrete $\mathrm{B} 30=30 \mathrm{MPa}$,

- Young's modulus = $34 \mathrm{GPa}$,

- Poisson's ratio $=0.2$,

- mass density $=2500 \mathrm{~kg} / \mathrm{m}^{3}$,

- damping coefficient $=0.04$ (a hysteretic damping is used),

The structure is subjected to a time loading applied at the center of a side wall (point $P 0$ in Figure 10). This loading and the boundary conditions of the structure are modeled and described in Figure 10 . 


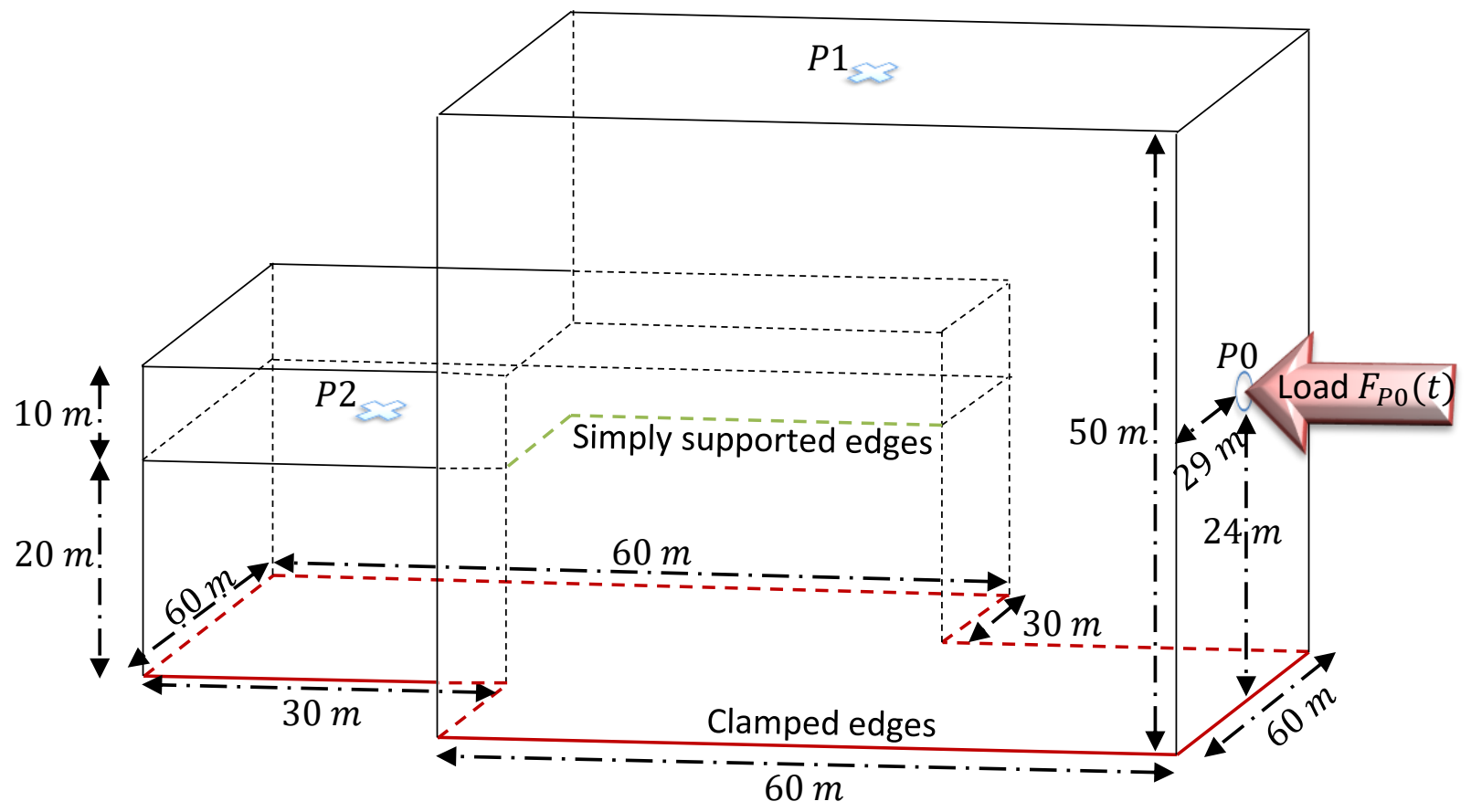

Figure 10: Geometry of civil engineering structure studied.

The versine force $F_{P 0}(t)$ with an amplitude of $1 \mathrm{MN}$ is defined in Figure 11.

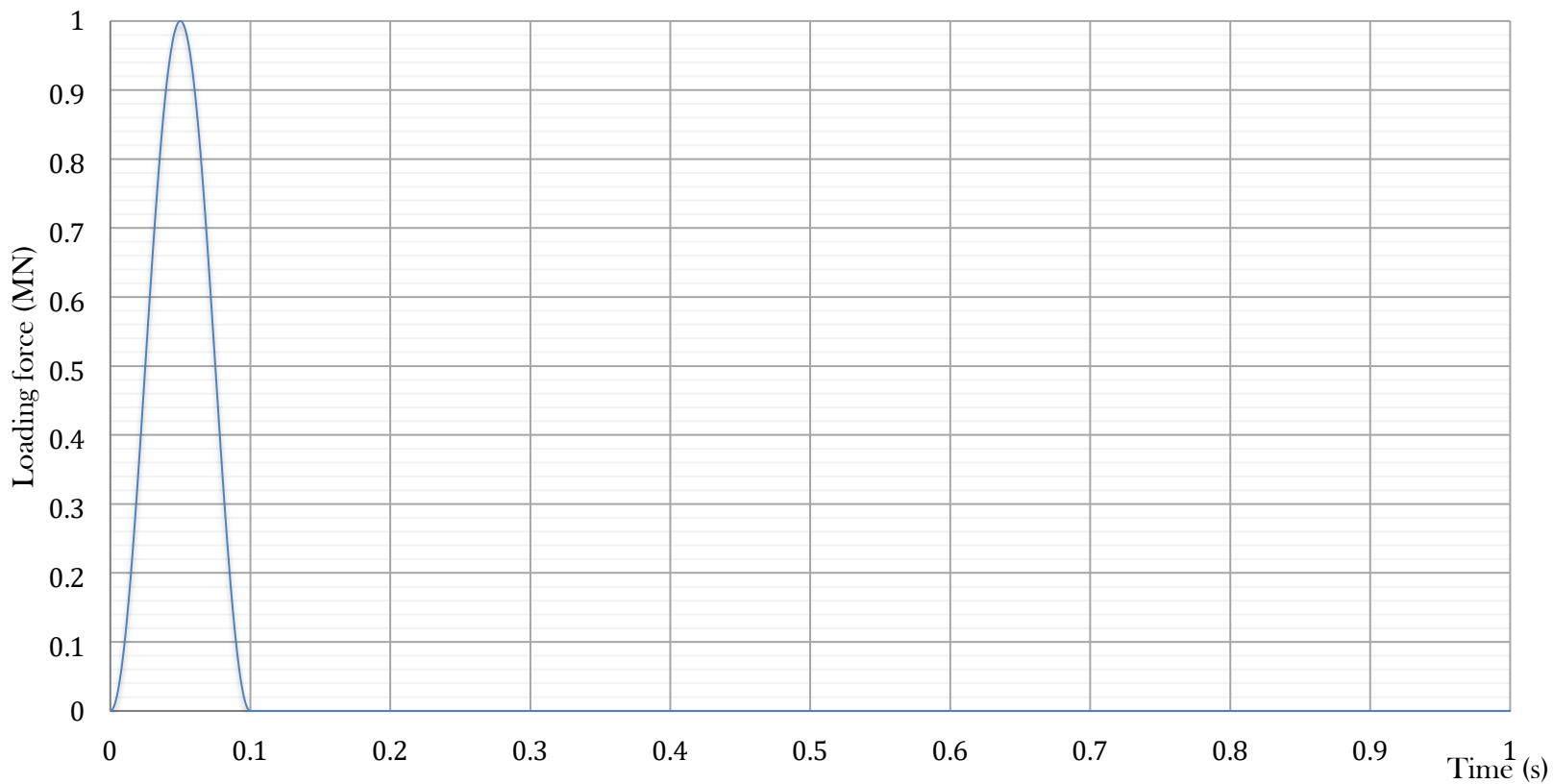

Figure 11: Time loading function.

Figure 12 describes the frequency content of the loading function applied. 


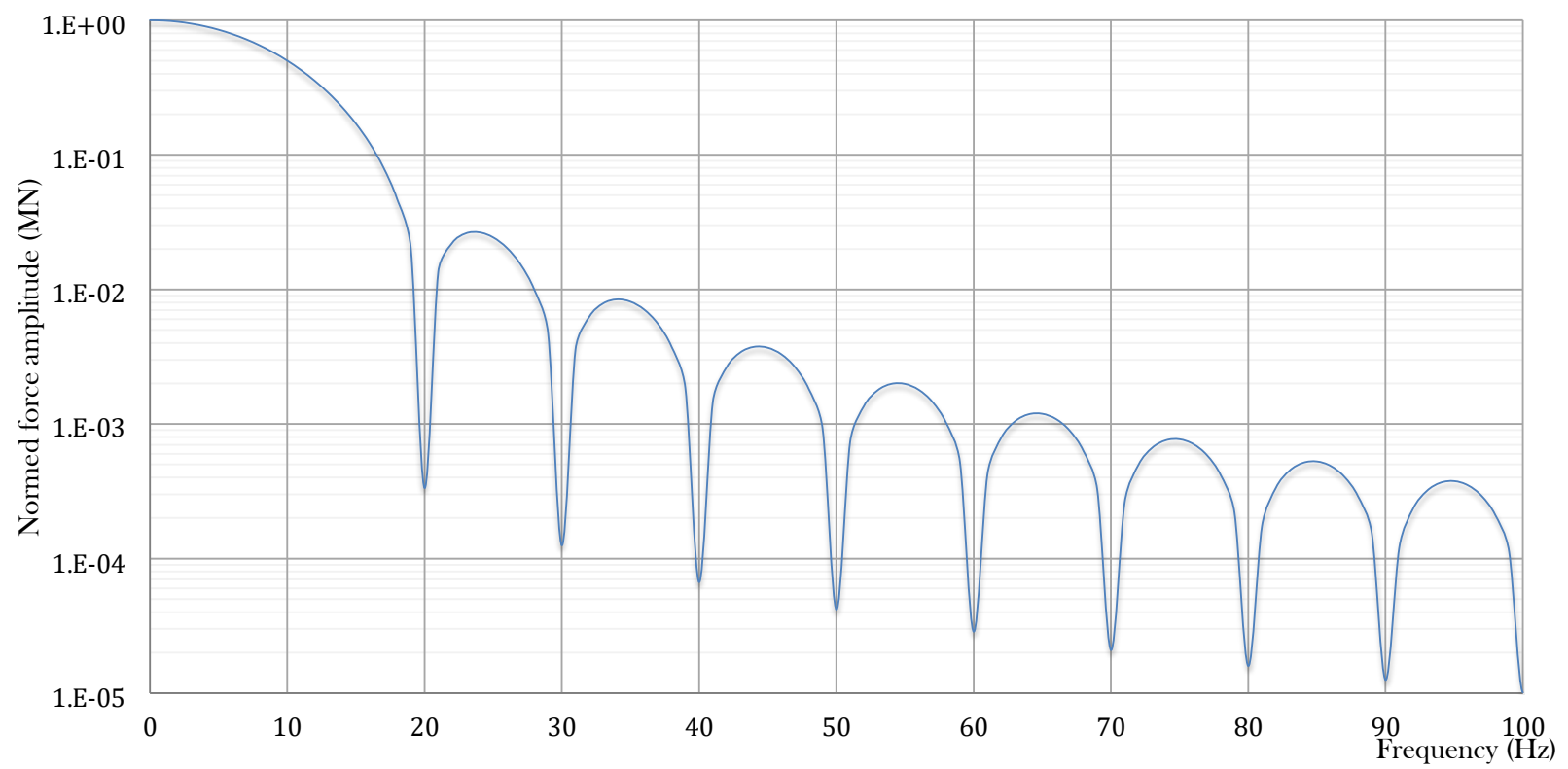

Figure 12: Frequency content of $F_{P 0}$ versine loading function.

Two hundred waves are sufficient to properly represent the frequency response [27]. Table 4 shows the VTCR frequency solution obtained at four different frequencies. A verification of the boundary conditions is done to check the quality of the solution.

Table 4: VTCR solutions of civil engineering example at four different frequencies. 


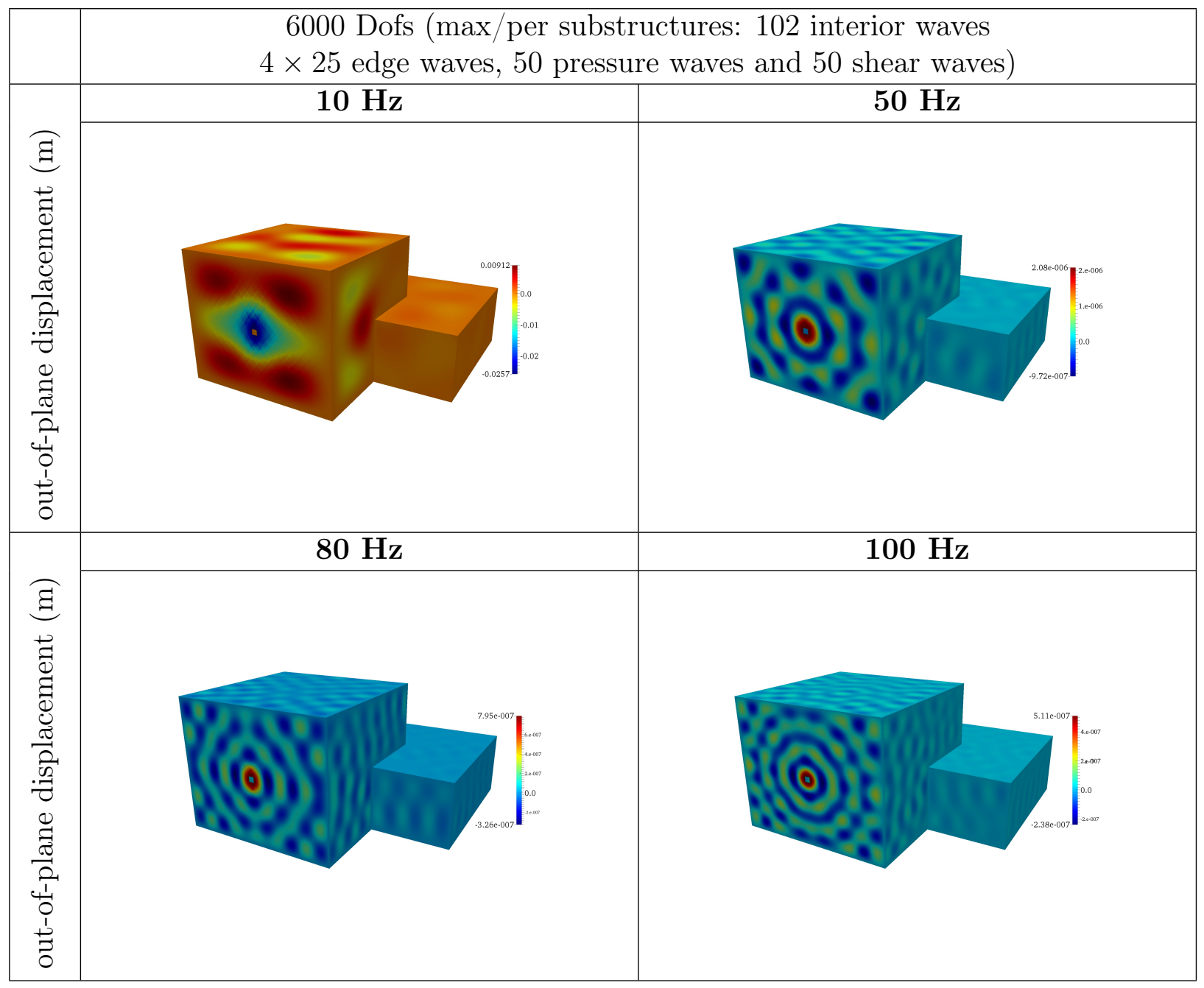

Figure 13 presents the results for the point $P 1$. Bandwidth is refined in this case studied with the $10 \%$ criterion based on the error between the reference VTCR frequency-by-frequency solution and the Padé approximants solution. In the case where the reference solution is not known, bandwidth refinement by dichotomy, as stated in paragraph 4.1, could be used. Here this bandwidth begins at $10 \mathrm{~Hz}$ and is initially refined to $4 \mathrm{~Hz}$. In this case, only 13 matrix inversions are enough to present good results on a frequency bandwidth of $100 \mathrm{~Hz}$. 


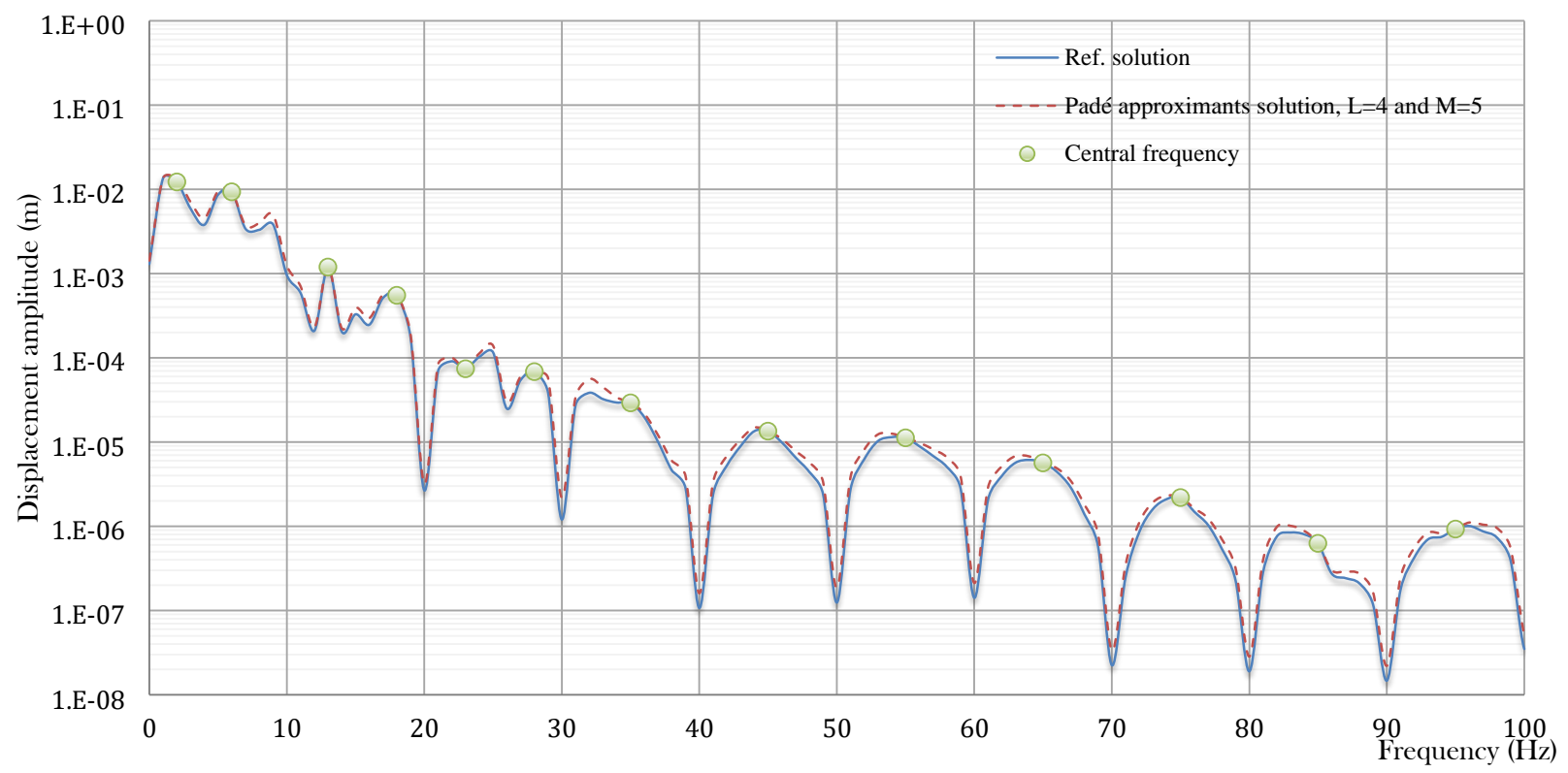

Figure 13: FRF over an adapted wide frequency bandwidth, with a standard criterion of $10 \%$, for point $P 1$.

Figure 14 shows the results for the point $P 2$ with the same previous bandwidth discretization. We can observe that the error can be higher than $10 \%$ on some frequencies. These errors are mainly due to the fact that the modal content of the floor at the point $P 2$ is slightly different than that of the area of the point $P 1$. Nevertheless, The error remains below $20 \%$ which is better than finite element calculations which one not refined enough.

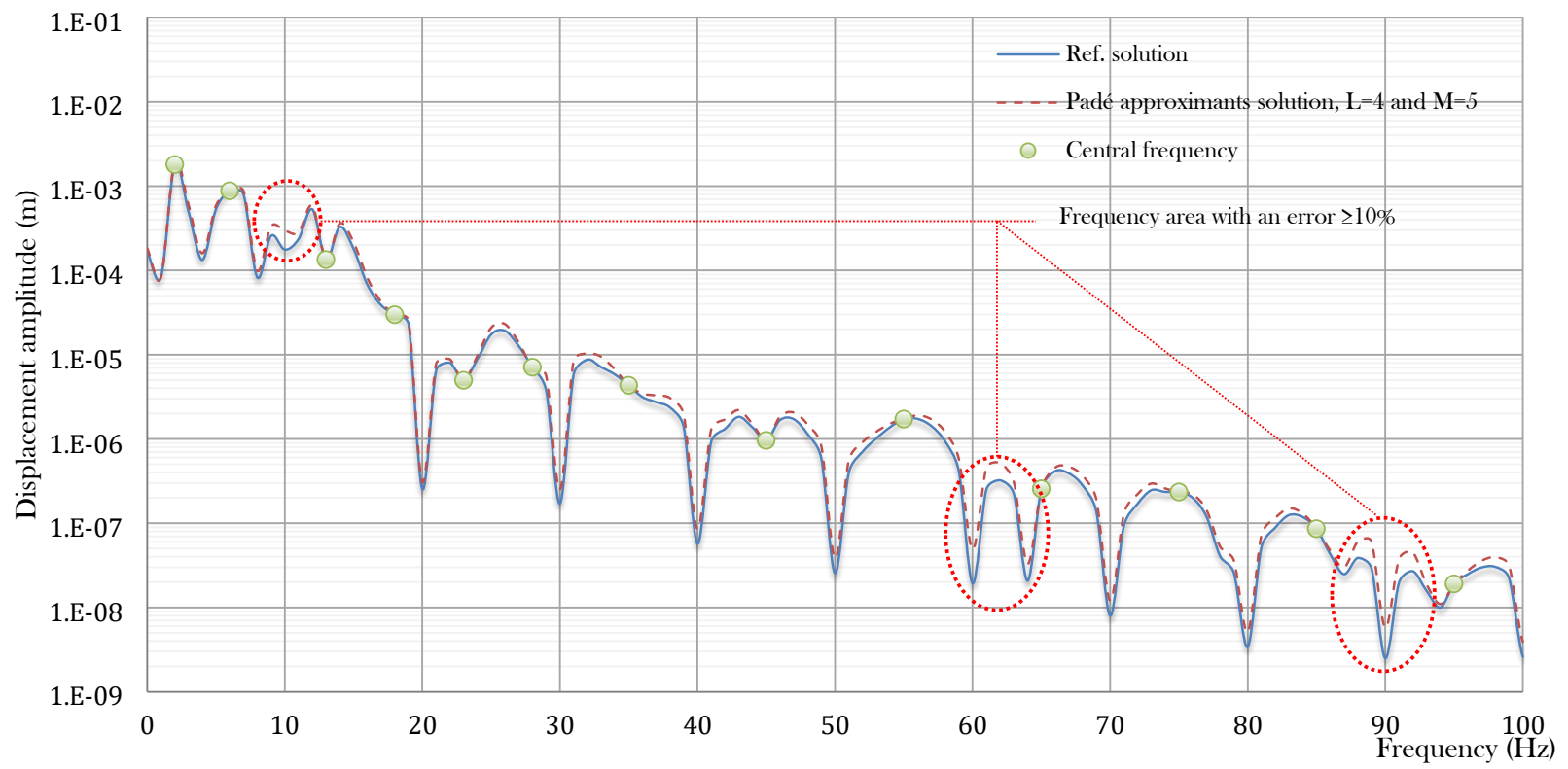

Figure 14: FRF over the previous adapted wide frequency band for point $P 2$. 
To go further in the analysis, it is possible to return to the time domain in order to analyze the impact of these errors on the response of the structure. Figure 15 shows the displacement at point $P 2$ of the floor. One can thus see that despite the $20 \%$ maximum error at some frequencies, the time displacement response is only slightly affected. The error in this example is less than $1 \mathrm{~mm}$.

The displacement response allows us to observe easily and graphically the low frequency content. It is recommended to use the acceleration response to focus on errors in medium frequencies ranges. So Figure 16 allows us to qualify errors with multi-frequency analysis in medium frequencies. One can see that the approximation error remains low ( $\leq 4 \mathrm{~g}$ in our case).

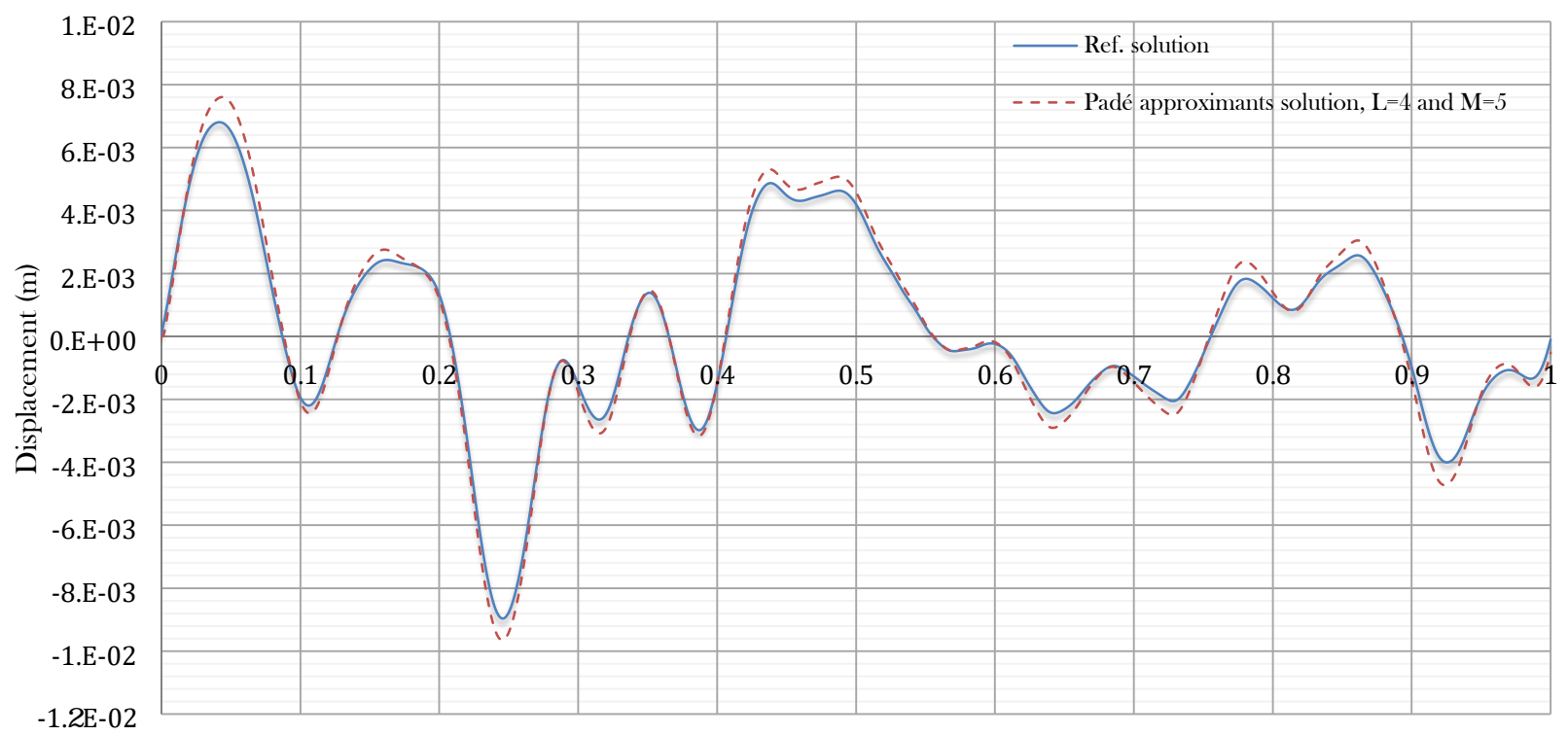

Time (s)

Figure 15: Time displacement response over the previous adapted wide frequency band for the point $P 2$. 


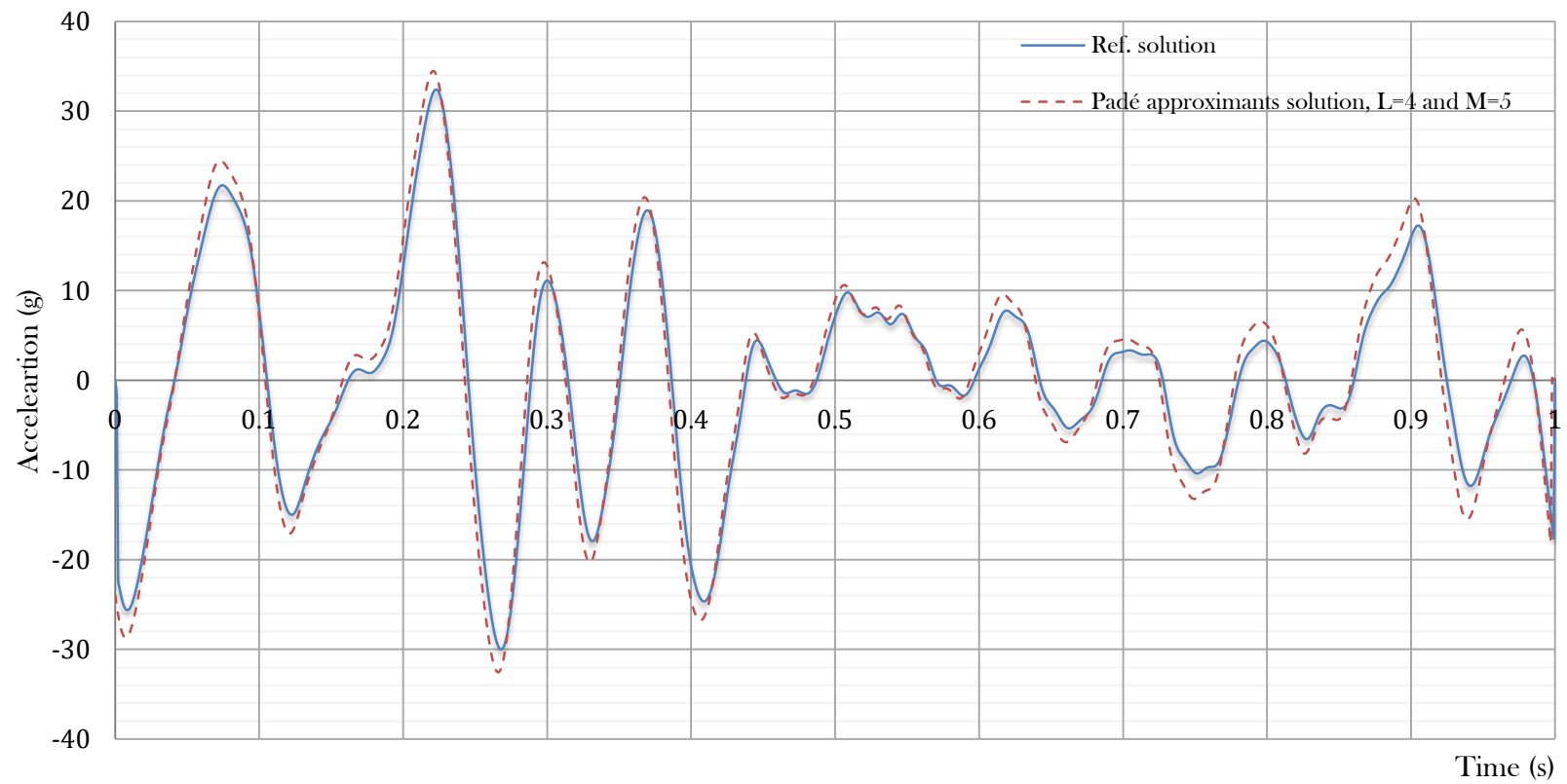

Figure 16: Time acceleration response for the point $P 2$.

To conclude, in this application a multi-frequency analysis with Padé's approximants provides good results due to the fact that the modal content of the studied structure is known. In this case, the frequency bands could be adapted to the modal content. Nevertheless, it is interesting to note that in a first approach, the proposed adapted multi-frequency analysis allows to describe an approximate response of the structure with a computational cost reduced by approximately $30 \%$. In the case studied, the computational cost for a frequency-by-frequency resolution is around $105 \mathrm{~min}$.

If the modal content is not known, the same analysis can be done by choosing first arbitrary frequences. In a second step the bandwidth can be divided by two and an error between the two solutions computed. If this error seems not acceptable the bandwidth can be divided by two again and the news error computed. This approach can be done until the differences between two solutions is assumed acceptable.

\section{Conclusions}

In this paper, a new way has been presented for the treatment of impact problems on civil engineering structures, as well as the determination of the shaking induced on industrial structures. The approach is based on a FFT/VTCR/IFFT methodology coupled with a multi-frequency resolution based on use of Padé approximants.

The previous FFT/VTCR/IFFT methodology, based on a frequency-by-frequency resolution, has already been shown to be an accurate way for solving transient dynamic impact problems over a low and medium frequency range. However, it could exhibit a loss of efficiency in the case of large spectrum loadings. The multi-frequency resolution developed in this paper, based on a Padé expansion of the VTCR problem in the frequency domain, can be seen as a large band analysis methodology. The main conclusions about this approach are:

- the Padé expansion gives accurate results on plate assemblies, but requires either the modal 
content of the structure to be a priori known, or to proceed by dichotomy with an error criterion as proposed in this article;

- to overcome this issue, a multi-point approach based on a bandwidth adaptation related to a convergence criterion can be used. This methodology provides good results with insignificant errors on FRFs and on time displacement and acceleration responses. However, the results depend on the eigenmodes excited by the input load, and more particularly by specific modes such as floor modes or wall modes which may vary between the studied area and the area used to define the frequency bandwidth;

- application of the Padé expansion is efficient and accurate on plate assemblies compared to the previous frequency-by-frequency approach. In the case studied in this article, the time saving was estimated to be nearly $30 \%$ (32 min) compared to a classic frequency-by-frequency resolution;

- however, application of the proposed strategy to structures involving shell substructures seems to be more problematic. Indeed the method requires the calculation of successive derivatives of the VTCR terms with respect to the frequency what is numerically a tough task in the implementation for shell substructures VTCR shape functions. Further developments should be carried out in order to adapt the Padé approximants approach to structures including shell parts.

\section{Acknowledgements}

This work has been funded by AREVA. The authors would like to thank N. Moussallam for hosting this research and his valuable comments.

\section{References}

[1] G. Hervé, F. Gatuingt, A. Ibrahimbegovic, On numerical implementation of a coupled rate dependent damage-plasticity constitutive model for concrete in application to high-rate dynamics, Engineering Computations 22 (5/6) (2005) 583-604.

[2] G. Hervé, C. Rouzaud, F. Barré, E. Secourgeon, Optimizing the analysis of airplane crash induced spectra by means of generic airplane methodology, in: Proceedings of the 22nd SMiRT, San Francisco, USA, 2013.

[3] J. Wolf, K. Bucher, P. Skrikerud, Response of equipment subjected to aircraft impact, Nuclear Engineering and Design 47 (1) (1976) 169-193.

[4] E. Jacquelin, J.-P. Lainé, A. Bennani, M. Massenzio, A modelling of an impacted structure based on constraint modes, Journal of sound and vibration 301 (3) (2007) 789-802.

[5] I. Babuška, F. Ihlenburg, E. T. Paik, S. A. Sauter, A generalized finite element method for solving the helmholtz equation in two dimensions with minimal pollution, Computer Methods in Applied Mechanics and Engineering 128 (3) (1995) 325-359. 
[6] S. Marburg, Six boundary elements per wavelength: is that enough?, Journal of Computational Acoustics 10 (01) (2002) 25-51.

[7] M. Kassem, C. Soize, L. Gagliardini, Structural partitioning of complex structures in the mediumfrequency range. an application to an automotive vehicle, Journal of Sound and Vibration 330 (5) (2011) 937-946.

[8] L. Geng, X.-Z. Zhang, C.-X. Bi, Reconstruction of transient vibration and sound radiation of an impacted plate using time domain plane wave superposition method, Journal of Sound and Vibration 344 (2015) 114-125.

[9] D. Zhu, H. Chen, X. Kong, W. Zhang, A hybrid finite element-energy finite element method for mid-frequency vibrations of built-up structures under multi-distributed loadings, Journal of Sound and Vibration 333 (22) (2014) 5723-5745.

[10] C. Rouzaud, F. Gatuingt, D. Dorival, G. Hervé, L. Kovalevsky, A new way for the simulation of the impact on reinforced concrete structures, Engineering Computations 32 (8) (2015) 2343-2382.

[11] H. Riou, P. Ladeveze, P. Rouch, Extension of the variational theory of complex rays to shells for medium-frequency vibrations, Journal of Sound and Vibration 272 (1) (2004) 341-360.

[12] P. Ladevèze, L. Arnaud, P. Rouch, C. Blanzé, The variational theory of complex rays for the calculation of medium-frequency vibrations, Engineering Computations 18 (2001) 193-214.

[13] P. Rouch, P. Ladevèze, The variational theory of complex rays: a predictive tool for mediumfrequency vibrations, Computer Methods in Applied Mechanics and Engineering 192 (28) (2003) 3301-3315.

[14] P. Rouch, C. Blanzé, Vibrational analysis of structures with stochastic interfaces in the mediumfrequency range: Experimental validation on a touch screen, Journal of Sound and Vibration 333 (6) (2014) 1612-1628.

[15] P. Ladevèze, M. Chevreuil, A new computational method for transient dynamics including the low-and the medium-frequency ranges, International Journal for Numerical Methods in Engineering 64 (4) (2005) 503-527.

[16] H. Padé, Sur la représentation approchée d'une fonction par des fractions rationnelles (The approximate representation of a function by rational fractions), no. 740, Gauthier-Villars et fils, 1892.

[17] R. Rumpler, P. Göransson, J.-F. Deü, A finite element approach combining a reduced-order system, padé approximants, and an adaptive frequency windowing for fast multi-frequency solution of poro-acoustic problems, International Journal for Numerical Methods in Engineering 97 (10) (2014) 759-784.

[18] C. Vanmaele, D. Vandepitte, W. Desmet, An efficient wave based prediction technique for plate bending vibrations, Computer methods in applied mechanics and engineering 196 (33) (2007) $3178-3189$. 
[19] L. Kovalesky, P. Gosselet, Optimized approximation space for trefftz-discontinuous galerkin methods, application to the variational theory of complex rays, International Journal for Numerical Methods in Engineering.

[20] P. Avery, C. Farhat, G. Reese, Fast frequency sweep computations using a multi-point padébased reconstruction method and an efficient iterative solver, International Journal for Numerical Methods in Engineering 69 (13) (2007) 2848-2875.

[21] S. A. Orszag, C. Bender, Advanced mathematical methods for scientists and engineers, Mac Graw Hill.

[22] G. Baker Jr, P. Graves-Morris, Padé approximants, Vol. 59, Cambridge University Press, 1996.

[23] B. Cochelin, N. Damil, M. Potier-Ferry, Méthode asymptotique numérique: une technique de résolution des équations non linéaires, Hermes Science Publishing, Paris, Londres (2007) 121135.

[24] R. Djellouli, C. Farhat, R. Tezaur, A fast method for solving acoustic scattering problems in frequency bands, Journal of Computational Physics 168 (2) (2001) 412-432.

[25] Cast3m, http://www-cast3m.cea.fr/ (1980).

[26] O.-N.-R. Office for Nuclear Regulation, Safety assessment principles (saps), Tech. rep., Technical Assessment Guides (TAGs) (2014).

[27] C. Rouzaud, Ébranlement de structures en béton armé soumises à un phénomène transitoire (shaking of reinforced concrete structures submitted to transient dynamic phenomenon), Ph.D. thesis, Thèse de doctorat, Université Paris-Saclay, ENS Cachan (2015). 